\title{
Attachment Preferences in Diverse Collective Problem-Solving Networks and Systemic Performance
}

Acknowledgements: We would like to thank the Dartmouth Sociology Junior Faculty Reading Group, participants of the International Conference on Computational Social Science $\left(\mathrm{IC}^{2} \mathrm{~S}^{2}\right)$, and Christopher Hanusa who all provided very insightful and thoughtful comments on various versions of this article. All mistakes are our own.

Funding: This project was funded by the National Science Foundation (NSF) grant \#2022395.

\author{
Charles J. Gomez ${ }^{1}$ \\ Queens College, The City University of New York \\ Department of Sociology \\ Antonio D. Sirianni \\ Department of Sociology \\ Dartmouth College \\ Launy Schweiger \\ Queens College, City University of New York
}

\begin{abstract}
Collective problem-solving networks are common in modern life. They often benefit from having diverse members with complementary skills and perspectives, however the benefits of information exchange and synthesis among them may be squandered if they self-select away from diverse counterparts and towards homogeneous groups or perceived competency. Building on the extensive tradition of "exploration and exploitation" agent-based models (ABMs), we initialize communicative networks with diverse groups of agents who solve a complex problem represented by Kauffman's NK problem space. We compare three types of ABMs, where the initial network and agent setups are identical but differentiated by agents' attachment proclivities: (1) diversityseeking networks, where agents prefer ties with different-agents; (2) homophily-seeking networks, where agents prefer ties with similar-agents; and (3) merit-seeking networks, where agents prefer ties with agents who have found better solutions. We find that diversity-seeking networks perform well because diversity promotes more exploration for solutions, but it also fosters network structures that disseminate these higher quality solutions more effectively than merit-seeking and homophily-seeking networks.
\end{abstract}

Keywords: agent-based models; collective-problem solving; communicative networks; diversity.

${ }^{1}$ Charles J. Gomez

City University of New York, Queens College

Department of Sociology

252-DD Powdermaker Hall

65-30 Kissena Boulevard,

New York, New York, 11367-1597

charles.gomez@qc.cuny.edu 


\section{Introduction}

2 Diverse collective problem-solving networks are common in modern life. These setups often

3 involve diverse sets of people collectively solving a complex problem that no one could solve on

4 their own (Boroomand \& Smaldino, 2021; den Hamer \& Frenken, 2020; Gomez \& Lazer, 2019;

5 Hong \& Page, 2004; Lazer \& Friedman, 2007; March, 1991; Shugars, 2021). While research shows

6 that diversity improves problem-solving, it typically assumes patterns of interactions between

7 diverse alters are fixed or even imposed by an organizing entity. An overlooked aspect of diversity

8 is how individuals choose to interact with different alters in problem-solving settings. When people

9 choose whom they interact with, a strong propensity to self-select into homogeneous groups or to

10 select based purely on perceived competency often exists. So, the problem-solving benefits that

11 emerge from a truly integrative diverse setting - one that involves the information exchange and

12 synthesis of differing perspectives - may be squandered as people self-select away from diverse

13 counterparts (Boone \& Hendriks, 2009). Indeed, the focus on the diversity of composition, or how

14 much variation exists within the group regarding the trait of interest, may come at the expense of

15 whether individuals interact with different alters at all. Alternatively, the search process for diverse

16 counterparts itself may have unintended and adverse outcomes, as it could inhibit the exploration

17 for better solutions. Given the role of self-organization (Ren et al., 2016), decentralized

18 communicative network structures (Rulke \& Galaskiewicz, 2000), and the constant churn and

19 mixing of individual perspectives (Shugars, 2021; Tyre \& Von Hippel, 1997) in fully leveraging

20 the benefits of diversity, the examination of attachment proclivities towards (or away from)

21 diversity and their consequences remains a surprising oversight in our current understanding.

While it is tempting to draw obvious conclusions about the role of diversity preference in

23 collective problem solving, the influence of individual preferences on the variation, exchange,

24 development, and dissemination is far from obvious. Diversity preference might lead to the more 
1 efficient spread of a wider range of solutions across a more connected organization. Diversity

2 preference also might undermine the attention paid to superior solutions, inhibit the formation of

3 problem-solving clusters, and stifle innovation by spreading early solutions across group-

4 boundaries too quickly.

To better understand why seeking diversity yields better results in collective problem

6 solving outcomes, we build on the extensive tradition of using computational experiments in the

7 form of agent-based models (ABMs), which are frequently used to model these settings (Arentze

8 et al., 2013; Banisch et al., 2012; De Marchi \& Page, 2014; den Hamer \& Frenken, 2020; Fang et

9 al., 2010; Fioretti, 2013; Gomez \& Lazer, 2019; Lazer \& Friedman, 2007; Shi et al., 2017; Smith

$10 \&$ Conrey, 2007). ABMs are helpful in showing how the behaviors and interactions of independent

11 agents generate collective outcomes. Following from the traditional "exploration and exploitation"

12 modelling setup (March, 1991), diverse sects of agents are randomly distributed in a network and

13 solve a complex problem metaphorically represented in our model with Kauffman's NK problem

14 space (Kauffman, 1993), an abstract mathematical space conceptually resembling a mountain

15 range. Agents "explore" this space seeking higher and higher peaks that reflect better scores. The

16 NK-paradigm has been widely used in the ABM and collective-problem solving literatures as a

17 theoretical model for how individuals and groups solve complex problems (Csaszar, 2018;

18 Levinthal, 1997; Rivkin, 2000; Rivkin \& Siggelkow, 2003; Siggelkow \& Rivkin, 2009).

19 Diversity is operationalized here as the ability to see problems through different lenses

20 because of differing backgrounds, experiences, or abilities, as opposed to purely demographic-

21 diversity We are interested in how diversity-seeking agents can improve collective outcomes. Our

22 models vary agents' proclivities to affiliate with dissimilar agents who can explore the problem

23 space in a different way. Information sharing between diverse agents is shown to reveal new 
1 pathways to better solutions that were not obvious if they only communicated with like agents

2 (Boroomand \& Smaldino, 2021; Gomez \& Lazer, 2019; Hong \& Page, 2004; Shugars, 2021). To

3 test the effects of preferences towards diversity, initial networks are set up in the exact same way

4 (e.g., NK space, distribution of agents, starting solution, etc.) but are populated with agents guided

5 by three different preferential attachment behaviors: one where agents prefer ties with different-

6 agents (diversity-seeking), a second where agents prefer ties with similar-agents (homophily-

7 seeking), and a third where agents connect with agents who have found better solutions (merit-

8 seeking). We then compare the relative performance — defined as the average NK score of agents

9 in the network - of each attachment behavior in simulations that permit local tie searches with

10 simulations that instead permit global searches throughout the network.

11 Diversity-seeking agents generally performs well in collective problem-solving networks,

12 but the reason why is surprising. While diversity promotes more exploration for solutions, it also

13 fosters network structures that better disseminate solutions. In addition, whether agents search

14 locally or globally for connections, diversity-seeking networks consistently produce the highest

15 quality solutions. So, diversity seeking does well not only because it promotes exploration for the

16 best solutions, but because it also fosters network structures that disseminate these higher quality

17 solutions more effectively. These networks have lower levels of clustering and transitivity,

18 facilitating more efficient information transmission. By contrast, merit-seeking networks perform

19 better in the short-run, but quickly adopt and circulate mediocre solutions in the short-run, while

20 homophily-seeking networks perform the worst across all timescales. 


\section{2. Motivation}

\section{2.1. Cognitive Diversity in Collective Problem Solving}

3 While diversity is broadly defined along various forms of categorical identity (e.g., gender, race,

4 ethnicity, class, party affiliation, discipline, occupation, etc.), much of what undergirds diversity's

5 benefits in problem-solving or deliberative settings is the diversity in cognition that identity

6 diversity brings. This is the ability to see problems through different lenses because of differing

7 backgrounds, experiences, or abilities (Mello \& Rentsch, 2015; Page, 2008). Research consistently

8 shows that cognitive diversity vastly improves systemic outcomes and solutions when it is both

9 complementary and salient to the problem at hand (Boroomand \& Smaldino, 2021; Lamm et al.,

10 2012; Mello \& Rentsch, 2015; Mitchell et al., 2009; Olson et al., 2007; Page, 2007, 2008; Shugars,

$112021)$.

Mello and Rentsch (2015) provide a thorough review of the definitions of cognitive

13 diversity and its importance in groups. Cognitive diversity may refer to personality (Harrison et

14 al., 2002), knowledge and skills (Martins et al., 2013), differences in thinking styles (Shin et al.,

15 2012) or variation in attitudes, beliefs, and values (Tegarden et al., 2009). Mello and Rentsch

16 (2015) place forms of cognitive-diversity into three categories: trait-based (personalities, problem-

17 solving styles, learning-solving styles), developmental (values), and acquired (informational

diversity). The value of diversity along these lines varies as a function of how diversity is measured

19 and the specific details of the task at hand.

20 The benefits of cognitive diversity along the lines of demographic attributes such as race

21 or gender have been contrasted to the benefits of task relevant diversity. The research here is also

22 mixed, as differences along race or gender can prime negative-stereotyping within groups,

23 inhibiting performance, yet more task-relevant differences may come to be correlated with task-

24 irrelevant differences (Milliken \& Martins, 1996). There are also challenges to within group 
1 cohesiveness and communication within diverse teams that can undermine performance (Jackson,

2 1996). To the extent that larger social structures and culturally shared biases disproportionately

3 assign experiences and perspectives to people along the lines of task-irrelevant characteristics,

4 task-relevant diversity at the collective level may become correlated with task-irrelevant diversity.

5 Empirical work has highlighted the potential benefits of ethnic (McLeod et al., 1996) and gender

6 diversity (Campbell et al., 2013; Hoogendoorn et al., 2013). Age diversity has also been found to

7 be an important predictor of group performance in more complex tasks (Wegge et al., 2008).

8 While the relationship between diversity and performance has been well studied across

9 several different dimensions, this research emphasizes a diversity primarily along the lines of

10 group composition. The influence that diversity has on performance should itself be dependent on

11 the inclinations that individuals in these groups have towards working with dissimilar individuals.

\section{2.2. How Diversity Improves Collective Problem Solving.}

14 The benefits that can come from diverse groups of individuals working together are realized

15 through several different mechanisms. For example, diverse perspectives may generate

16 constructive disagreements (Fiol, 1994), structurally diverse teams bring together disparate fields

17 of knowledge (Cummings, 2004), and cognitively diverse teams have been found to have a higher

18 transactional memory, enabling them to better synthesize different perspectives into creative

19 solutions (Aggarwal \& Woolley, 2019). So, while a collaboration amongst plumbers and oboists

20 may not yield fruitful outcomes in furthering string theory, ventures between computer engineers

21 and business strategists may produce the next big start-up.

22 Diverse problem-solving groups can outperform more homogenous groups of high23 performing individuals (Boroomand \& Smaldino, 2021; Hong \& Page, 2001, 2004; Shugars, 24 2021). The collective benefit of a diverse set of tools and skills can be greater than the sum of their 
1 individual benefits, and a diverse set of perspectives can minimize collective error in problems of

2 prediction and estimation (Page, 2007). In homogenous groups, however, the "wisdom of crowds"

3 can be undermined by correlated judgment errors (Palley \& Soll, 2019).

In collaborative situations, however, the benefits of diversity may not be fully realized by

5 simply having a wide range of talented people. When a problem-solving venture requires people

6 to interact with and build off one another, how group members distribute their attention also 7 matters.

\subsection{How Network Structure Impacts Problem Solving}

10 In addition to membership diversity, patterns of connectivity between members can also shape

11 collective performance. When a set of collaborators is large enough to make simultaneous

12 interaction between all members impractical or infeasible, then the structure of interactions

13 between collaborators (i.e., their social network) will also influence group problem-solving ability

14 (Lazer \& Friedman, 2007). Network structure influences several important aspects of collective

15 problem solving: the distribution of information, the percolation of social influence, and the 16 generation of innovation.

17 Social network structure facilitates the array of information each actor encounters during a 18 collective problem-solving venture. Having a set of "weak ties" with disconnected and non19 redundant alters provides access to more novel information (Burt, 1995, 2004; Granovetter, 1973).

20 This may come at the expense of higher "bandwidth" connections with similarly connected alters

21 (Aral \& Van Alstyne, 2011). Otherwise, if the number of ties in a network is held equal, 22 information should diffuse most efficiently in networks that minimize clustering, redundant ties,

23 and the global network diameter. Certain network structures can also induce creativity and 24 innovative solutions. Uzzi and Spiro argue that creative networks characterized by interconnected 
1 pockets, rather than disjointed cliques or an undifferentiated structure, are conducive to success in

2 the theatre industry (2005). The performance of network structure may also vary over time:

3 inefficient networks can stall at first, but this suboptimality can motivate independent searches for

4 better solutions to problems (Khraisha \& Mantegna, 2020). More generally, simulation-based

5 work has shown similarly that cultural evolution and collective problem-solving is facilitated by

6 intermediate levels of population fragmentation and integration (Derex et al., 2019).

7 Connected individuals may influence one another in a way that undermines the "wisdom

8 of crowds" in collective assessment tasks (e.g., how many beans are in this jar?), as this induces

9 the errors of individuals to correlate rather than offset (Lorenz et al., 2011). However, networks

10 can be structured in a way that mitigates this source of error. Becker, et. al (2017) show that social

11 influence improves collective accuracy when networks are decentralized rather than centralized.

12 Networks are often characterized by their volume of local ties, which enhance closure and

13 are rich in "bonding" social capital, versus their volume of global ties, which reduce overall

14 network diameters and are rich in "bridging" capital (Burt, 2004; Putnam, 2000). Different social

15 network structures are better suited for different collective tasks. Information diffusion tasks may

16 be best facilitated by more "global" networks, creative processes are best facilitated by networks

17 that have local ties to nurture pockets of innovation and global ties to spread locally generated

18 successes, and localized networks that prevent poor insights and perceptions from spreading

19 globally are best for tasks of estimation and collective decision making.

\section{2.4. Homophily and Heterophily in Networks}

22 The network structure of interaction and communication between collaborators, at both a group

23 and collective level, is important for collective efficacy. For instance, within a given social

24 network, the benefits of diversity may be linked to the rates of interaction between different 
1 individuals. However, homophily, the tendency for similar individuals to affiliate, is one of the

2 most robust findings in the social sciences (M. McPherson et al., 2001). Homophily is not only

3 found with regards to socio-demographic characteristics, such as age, gender, and race, that are

4 not directly relevant to task performance, but is also found along the lines of educational attainment

5 (Skopek et al., 2011), emotional well-being (Chancellor et al., 2017), and personality (Noë et al., 6 2016).

7 Yet the ability of networked individuals to resist this tendency may be conducive to the 8 synthesis of diverse perspectives and the emergence of beneficial network structures. Even small 9 levels of preference for similar alters may lead to highly clustered and segregated communities 10 and clustered social networks (Henry et al., 2011; Schelling, 1969), and in simulations of problem11 solving on social networks, interaction between different members consistently improves 12 outcomes (Boroomand \& Smaldino, 2021; Gomez \& Lazer, 2019; Shugars, 2021). When networks 13 are not pre-configured to facilitate connections between different alters, diversity may be unlikely 14 to arise naturally.

15 Ultimately, the link between diversity and success relies on both the increased variation of 16 the ideas, perspectives, and abilities generated by a diverse group, and their subsequent mixing via 17 social interaction. While a diversity leviathan could arrange individuals into a prescribed 18 organizational structure that facilitates interaction between groups, in practice many of the 19 interactions between teammates and co-workers is determined from the bottom-up. The patterns 20 of interaction that might emerge naturally from the decisions of individuals deserve special 21 attention. In an emergent social network with a diverse set of members, a shared commitment to 22 forging connections with dissimilar others may be a key ingredient to collective success. Some 23 suggest, however, that purposefully inclusive diversity—where people actively seek out and 
1 engage with different people and ideas - can yield worse outcomes than setups that are already

2 diverse but predicated on an equality of opportunities, where people engage with others based on

3 performance and merit rather than the intrinsic value of diverse perspectives or abilities (Walton

4 et al., 2013). While this may hold true in a group where the structure of interactions is trivial or

5 uninteresting (i.e., in a group small enough where everyone interacts with one another), the

6 tradeoffs between the networks that emerge from merit-seeking and diversity-seeking individuals

7 remain unexplored.

\subsection{Emergent Network Structures and Collective Problem Solving}

10 The literature on diversity, network structure, and problem-solving so far has largely overlooked

11 the importance of how individuals choose to collaborate with one another when seeking solutions

12 to complex problems. There are three potential strategies that individuals could be expected to employ (or behavioral tendencies that individuals may unwittingly embrace) when deciding who

14 to learn from in a collective problem-solving endeavor ${ }^{1}$. First, they may choose to prioritize

15 interacting with agents who differ from them and have a different set of problem-solving abilities.

16 This follows the seminal work of Hong and Page (2004), and other literature that indicates the

17 benefits that come from (task-relevant) diversity in the collective problem-solving literature.

Another strategy is to seek out interacting with agents that are the same as them, or agents

19 that have the same set of problem-solving abilities. Homophily has been documented within both

20 formal and voluntary organizations (Kleinbaum et al., 2013; J. M. McPherson \& Smith-Lovin,

21 1987) and is a reasonable default expectation. Gomez and Lazer (2019) find that segregating ideas

\footnotetext{
${ }^{1}$ We assume that individuals in a network will not behave in a purely random or static fashion, and do not examine these as control conditions here, but instead focus on heuristics that agents might reasonably employ in an actual setting. We are interested in how agency effects organizational networks, as opposed to a purely structured or chaotic process.
} 
1 leads to better outcomes than integrating them; segregating ideas provides a buffer that allows new

2 potential pathways to better solutions manifest. Otherwise, too many diverse ideas presented all at

3 once initially leads systems to quickly coalesce on less optima solutions, cutting off these latent

4 pathways. Homophily along qualitative or discrete characteristics may also create networks of

5 loosely connected clusters, which have been shown to improve creative processes (Uzzi \& Spiro,

6 2005) and provide a balance between exploration and exploitation in collective-problem solving

7 networks (Fang et al., 2010).

8 The other strategy considered is a meritocratic heuristic. In this case, individual agents look

9 for those who have come up with the best solution to the problem so far. While this does not

10 necessarily preclude affiliations with agents of a different type, it also does not prioritize diversity

11 explicitly. In practice, policies and strategies that promote meritocracy may end up reinforcing

12 biases against workers from marginalized groups (Castilla, 2008; Castilla \& Benard, 2010). Even

13 if practiced in a way that is free of bias, meritocracy may be a more promising strategy at the

14 immediate and individual level of the agent but may not lead to outcomes that are optimal for the

15 long-term collective perspective. In other work examining the NK-space optimization problem, 16 networks where high-performers are imitated are only beneficial when the complexity of the

17 landscape is low and are prone to settling on local maxima prematurely (Fontanari \& Rodrigues, 18 2016).

A preference for meritocracy may lead to more "centralized networks," and early work on 20 teams suggests that centralized networks perform simple tasks more competently, and 21 decentralized networks perform complex tasks more competently (Leavitt, 1960). Later work has 22 shown that centralized networks perform best after a decentralized exploration process (Siggelkow $23 \&$ Levinthal, 2003), but the cumulative advantage processes that generate these networks limit 
1 performance on both the NK-space problem, and the complex real-world problems that they

2 imitate (C. Watts \& Gilbert, 2011). Centralized network structure fundamentally creates a form of

3 attentional inequality which can both prematurely narrow the attention of the network on a small

4 number of individuals, but quickly disseminate successful innovations. While these networks may

5 perform better when 'high-achievers' are at the center, this form of inequality still may undermine

6 collective performance in the long-run.

\subsection{Agent-Based Modeling: Simulation Approaches to Diversity, Network Structure, and} Collective-Problem Solving

10 The interacting dynamics of network formation and collective problem solving are too complex to

11 allow us to predict results intuitively, so we turn to an agent-based modeling (ABM) approach. An

$12 \mathrm{ABM}$ allows us to test the macro-level consequences of a large group of interacting actors who

13 behave in accordance with a pre-existing set of rules. The premise of agent-based modeling is that

14 social outcomes of interest are a function of interacting individuals, as opposed to interacting

15 variables (Macy \& Willer, 2002). The ABM approach has been championed by complexity 16 scientists (Miller \& Page, 2009) and computational social scientists seeking to build "bottom-up"

17 models of social behavior (Epstein \& Axtell, 1996).

The specific problem we examine combines lines of agent-based modeling research that

19 are each relevant to our question. ABMs have demonstrated the importance of individual cognition

20 and preferences on collective outcomes, such as how homophily and social influence can lead to

21 segregation (Schelling, 1969) or polarization (DellaPosta, 2020), how deferential norms can

22 generate stratified and hierarchical networks (Manzo \& Baldassarri, 2015) and facilitate group

23 cooperation (Sirianni, 2018), and how associative reasoning can lead to opposing coalitions of

24 norms and values in otherwise cohesive groups (Goldberg \& Stein, 2018). 
As agent-based models are frequently used as a model for collective problem solving, our model builds upon and extends the traditional "exploration and exploitation" model commonly

3 used in the literature, a tradition that extends to the Carnegie School of organization science

4 (March, 1991; March \& Simon, 1993). The NK landscape is a commonly used approach for the 5 exploration and exploitation problem (Csaszar, 2018). It is essentially an $N$-dimensional typology

6 with $K$-level complexity, each point in the landscape has a different payoff, and agent(s) navigating

7 the landscape try to locate the point that will maximize their payoff. ABMs have been used to 8 model fixed networks ability to solve explore-exploit problems on the NK landscape (Boroomand 9 \& Smaldino, 2021; den Hamer \& Frenken, 2020; Fang et al., 2010; Gomez \& Lazer, 2019; Lazer $10 \&$ Friedman, 2007), as well as other multi-agent coordination problems that use the NK landscape 11 (Chang \& Harrington Jr, 2006; Kollman et al., 2000).

\section{3. Analytic Approach}

14 We now introduce our approach, where evolving networks of individual agents with varying social 15 preferences collectively explore the NK landscape. We code our ABMs in $\mathrm{C}++$ and used $\mathbf{R}$ to 16 create our NK problem spaces, which are available at the Harvard Dataverse (DOI:XX.XXXX). 17 Our ABM setup consists of comparing the outcomes of three initially identical types of networks: 18 (1) networks comprised up of diversity-seeking agents, (2) networks comprised up of homophily19 seeking agents, and (3) networks comprised up of merit-seeking networks agents. Each network is 20 a directed network, so ties are not mutually shared.

At the start of each simulation, each of the three networks are set up in the exact same way:

22 Each network is populated with 100 agents of 50 "A-species" agents and 50 "B-species" agents 23 and are randomly distributed across in a torus network. Each agent in these torus network has six 24 to ties to its closest neighbors in the ring setup. Agents across the three networks are tasked with 
the exact same Kauffman's NK space $(1993)^{2}$. An NK space is a commonly used abstract

mathematical space that is more concretely conceived of as a mountain range, where agents

3 "search" the problem space for its peeks that reflect local and global solutions. In addition,

corresponding agents across the three network setups receive the same initial starting solution. In

other words, agent number 5 in the diversity-seeking network receives the same solution as agent

number 5 in the merit-seeking network and agent number 5 in the homophily-seeking network.

However, within each network, all 100 agents' solutions are unique and different to each other.

Thus, the only difference across the three network simulations and their setups is the tie proclivity

behavior of agents once the simulation begins.

\footnotetext{
${ }^{2}$ The $N$ in NK refers to an $N$-long bit string of 0 s and $1 \mathrm{~s}$ that is the address of each point in the space and the $K$ is a tunable parameter that determines how rugged the problem space is, where $K<N$. Low values of $K$ can be thought of as one large mountain where the highest peak is easily reachable by traversing the gradient. High values of $K$ can be thought of as a highly chaotic terrain where the highest peak is very difficult to reach and is often surrounded by numerous local optima that can trap agents. We found that using the traditional NK space setup to create different terrains with varying of $K$, ranging from simple landscapes of $K=1$ to highly difficult terrains of $K=10$, was a challenge. It was difficult to calibrate different values of $K$ corresponding to different degrees of ruggedness. In other words, above $K=3$, the terrain proved too difficult for most agents to find the global optimum. Instead, we took inspiration from the traditional NK setup with score masking and used a combination of sine functions and normal distributions to visually create rugged terrains corresponding to different values of $K$ that were tunable for our model. The random solutions in the space are operationalized as an array of fifteen $(N=15)$ random 0 s and $1 \mathrm{~s}$. The array (i.e., solution) is an "address" that reference one of the $2 \mathrm{~N}$ normalized scores in the NK problem space, where an array associated with a score of 1 reflects the global optimum in the space, or the best possible solution. We use an $N=15$, where the solutions are an array of fifteen bits of either $0 \mathrm{~s}$ or $1 \mathrm{~s}$. The $K$ corresponds to how rugged the NK space is. We set $K=5$ to create a complex problem space, where the global optimum is difficult to find. For any NK space, there are $2 \mathrm{~N}$ solutions that define the space, so for our parameters of $N=15$ and $K=5$, the solutions numerically range from ' 000000000000 ' (or 0 when converted from binary) to ' 111111111111111 ' (or $215=32,768$ solutions when converted from binary). The scores are normalized such that the global optimum (or best solution) has a value of 1 . In the Supplemental MaterialsSection B, we re-run our results presented here using different values of $K$ to test if our results hold across different difficulty landscapes, ranging from $K=1$ (i.e., an "easy" single large mountain) to $K=10$ (i.e., a "very difficult" chaotic terrain). Our overall findings hold across the various setups.
} 
A

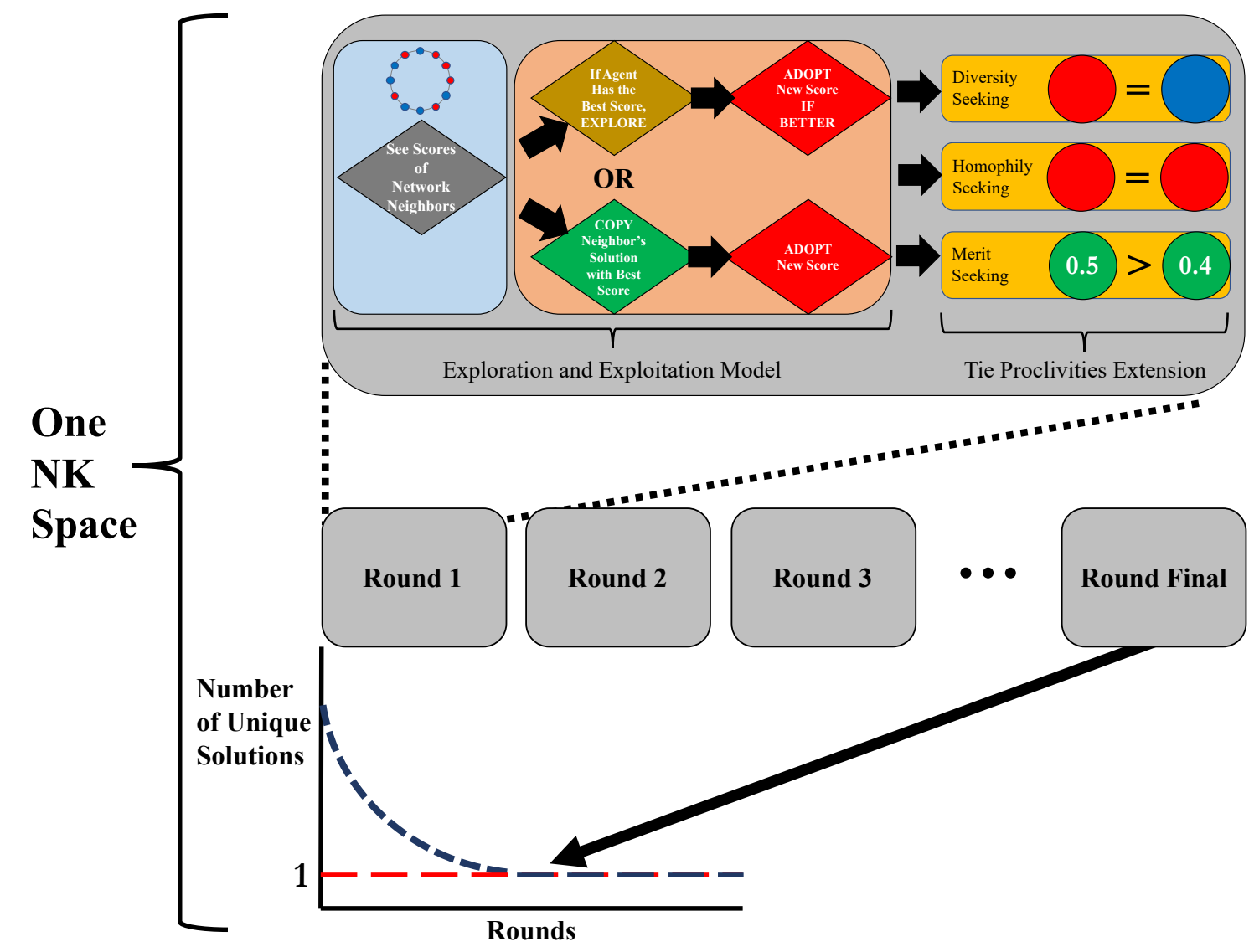

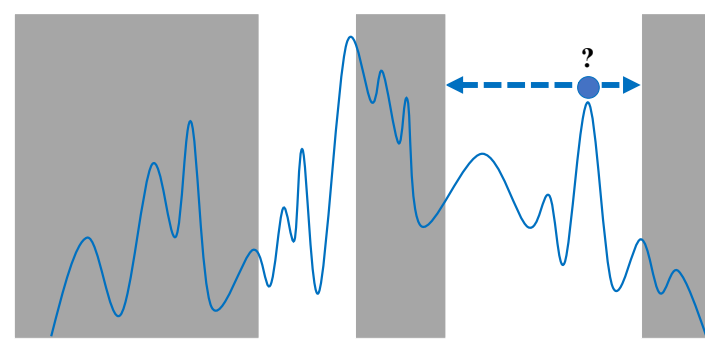
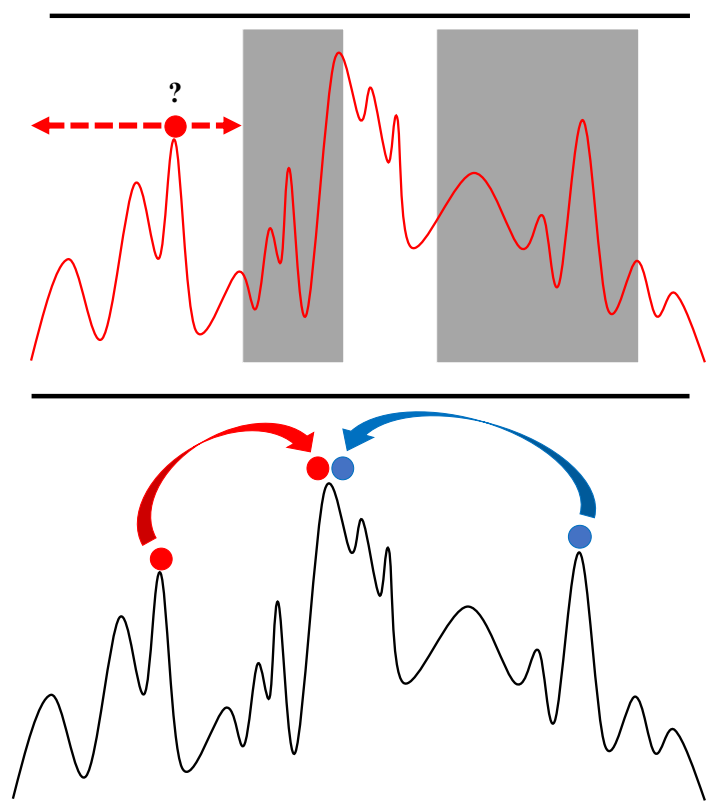

Figure 1. (A) The anatomy of a single NK space simulation. For a single NK space, the simulation runs for several rounds until the network reaches equilibrium (one unique solution in the network). Every round begins with the "exploration and exploitation" behavior and ends with our tie search extension, where each type of network engages in one of three tie-seeking behaviors. (B) How diverse agents traverse a complex NK space. Alone, agents can only see small sections of the wider problem space. However, when agents can communicate, in particular diverse agents who can see parts of the problem space that the others cannot, they can iteratively find higher and higher peaks (solutions). 
A single simulation run proceeds in iterative rounds until agents in the network achieve

equilibrium ${ }^{3}$. This occurs when all agents have the same score, and no unique solutions are being

3 introduced into the system. In Figure 1A, we visualize the anatomy of how the simulation works in each round, which is set up in two parts: (1) exploration and exploitation and (2) a search for new ties.

\subsection{Part One of a Round: Exploration and Exploitation}

8 The first part of a single round is an extension of the established Carnegie School "exploration and exploitation" setup modeled by Lazer and Friedman (2007), Gomez and Lazer (2019), den Hamer and Frenken (2020), Fang, et al. (2010), among others. Here, agents look at their six connections and if a neighboring agent has a better score, they then copy their neighbor's better NK solution. However, if the agent has the best solution among its neighbors, the agent then "explores" the NK problem space.

Agents "explore" by manipulating their solution in a way unique to their "species." Figure

15 1B conceptualizes how agents navigate a complex NK problem space, in particular diverse agents. able to see a small section of the NK space (the "darker" regions are areas that are inaccessible to that particular species of agent) ${ }^{4}$. As such, not only are agents not able to see the highest peak (i.e.,

\footnotetext{
3 We limit the simulations to 20 rounds, as all mean metrics of both solution-quality and network structure have converged to stable values by this point in time. While progressively smaller changes may occur in some metrics past round 20 (all networks will slowly converge to the optimal solution given enough time), the average rate of improvement becomes qualitatively indistinguishable from zero at this point.

${ }^{4}$ To ensure this and that any one specific search heuristic that agents use is not necessarily driving our results, we sample from four possible heuristics for any given NK space, outlined in the Supplemental Materials-Section A. As such, species "A" and "B" are merely placeholders. The search heuristic that A species and B species agents are endowed with at the beginning of each $\mathrm{NK}$ space is a random pairing of two different heuristics from the four heuristics, allowing for a total of six unique heuristic combinations (i.e., the same two heuristics are not never selected in the same NK space). For each endowed heuristic that an A species or B species agent has, the agent slightly alters its solution based on its assigned heuristic and then looks up the score of this mutated solution. If the agent finds a
} 
1 the global optimum of the NK space) on their own, they are also unable to access other regions of

2 the space that they could explore otherwise and settle prematurely. However, if diverse agents are

3 allowed to see the solutions of their different neighbor (the "exploitation" phase described earlier),

4 agents can not only see sections of the NK space that they could not see before, but agents can

5 now reach once inaccessible spaces, leading to higher and higher peaks (Gomez \& Lazer, 2019).

6 Our operationalization of diversity follows from Gomez and Lazer (2019) and their notion

7 of cognitive diversity as a "diversity of ability." (Thus, our ABMs are populated by two species of

8 agents, "A species" and "B species" in equal numbers.) Here, what distinguishes A agents from B

9 agents is that each species of agents manipulates their NK solution in some unique way that is

10 ultimately myopic but does not provide a tactical advantage over the other species of agent, so A

11 agents have no inherent advantage over B agents, and vice-versa. (How each agent manipulates

12 their NK solution to explore the NK space is explained in the Supplemental Materials-Section A.)

13 What is critical to our simulation is that agents should search the NK space in a mutually exclusive

14 way. In other words, the odds that both agents end up with the same exact solution after

15 manipulating the same initial solution is low, allowing for new pathways to open in the NK space.

16 Agents myopically climb the problem space in search of higher peaks in each round, reflecting

17 better solutions, but the terrain is such that it is quite easy for agents to become "stuck" in valleys

18 where local solutions may mask even better solutions elsewhere.

\section{3.2. Part Two of a Round: Searching for New Ties}

21 After the first "exploration and exploitation" part of the round is complete, the second part of the

22 round begins. Now agents in these networks are guided by one of three tie searching heuristics.

\footnotetext{
better solution after the manipulation, the agent then adopts this new solution as its own. Otherwise, the agent keeps
} the solution that it already had. 
1 This is our main treatment variable and our extension to the standard "exploration and exploitation" model, where agents in the networks choose new ties based on their proclivities.

In diversity-seeking networks, each agent flags each of their same-species connections for replacement ${ }^{5}$. They are then randomly assigned connections to new agents. If the ties are to agents that are different species to them, the agent keeps the connection. Otherwise, the agent rejects the new tie and retains its original tie. As such, these new random ties have no proclivity towards similar or dissimilar agents, so a search for more diverse ties may fail, but since the agent rejects

any new ties to similar agents, its connections will not become less diverse.

The search for random ties works in the same way for homophily-seeking networks and merit-seeking networks. For homophily-seeking networks, the inclusion criterion is the opposite to diversity-seeking: Agents must be the same species to them for the agents to keep the connection. For merit-seeking network, however, agents instead flag agents whose solutions are inferior to their current solution for replacement (i.e., the same solution or better solution).

We also define the pool of potential ties in one of two ways: the ties are randomly sourced "locally" or from the entire "global" network. By "local," new random ties are selected from agents' local neighborhood in the network: the pool of connections of their neighbors' neighbors ${ }^{6}$.

\footnotetext{
${ }^{5}$ As a robustness check, in the Supplemental Materials-Section B, we also test lower thresholds for agents to stop tie searching. Instead of redirecting ties until all six are similar (homophily), dissimilar (diversity), or superior (merit) ties are identified, the threshold is lowered in one set of simulations to five of six and in another to four of six ties. For example, for agents in merit-seeking networks, when their neighbor criterion is lowered to four agents, agents will stop looking for new ties when four of their neighbors have the same score as them or better score than them. We plot the performance across all three neighborhood thresholds (i.e., four neighbors, five neighbors, and the current criterion plotted here, six neighbors), as well as for problem space difficulty with $\mathrm{K}=1,3,5$, and 10 . Across these various parameters, our findings remain steadfast.

${ }^{6}$ Local tie searches also reflect how tie forms in real-world contexts. First, for many large teams or organizations, people often only have knowledge of the performance and characteristics of those close to them in the workplace, not the entire directory. This aligns with empirical research showing that connections in firms are largely predicted by induced local structure, such as departmental divisions (de Oliveira Maciel \& Netto, 2020). We also expect that local knowledge would likely come from intermediary connections. Second, this rewards network heuristics that are rich in bridging-capital and low in network clustering in a way that is consistent with the social capital literature: Individuals with non-redundant ties have access to more information about organizational members and can find target connections more quickly (Burt, 2004).
} 
1 And by "global," new random ties are selected randomly from the entire network, rather than just

2 from agents' local neighborhoods in the network.

3 By comparing the outcomes of the simulation setups where potential ties are either local or

4 global, we can compare the features of the emergent structure of this network with its more

5 localized counterpart and compare the quality of the solutions produced across the three network

6 setups. This is because allowing for access to the entire network (i.e., global sampling of ties)

7 improves the rate of information flow in the entire network, as areas previously unconnected can

8 see one another making information spread farther in the network. (This works in much the same

9 way as the "small world" phenomenon of Watts \& Strogatz, 1998.) When information velocity is

10 faster, the networks achieve equilibrium much faster, as earlier access to relatively better solutions

11 tends to bring about equilibrium faster (see Lazer \& Friedman, 2007). As such, the structure of the

12 across the three network setups - diversity-seeking, merit-seeking, and homophily-seeking

13 networks - should be relatively similar to one another. And if the network structure is mostly

14 similar across setups with these global experiments, any deviations with the results from their

15 corresponding local tie search network simulations - in other words, comparing the results from

16 local and global diversity-seeking networks, comparing the results from local and global merit-

17 seeking networks, and finally comparing the results from local and global homophily-seeking

18 networks - can be attributed to the emergent structure and not the impact of the exposure to 19 diversity.

20 Prior work has explored the differences between localized and global network structures 21 and their implications of for the dissemination and production of knowledge (see Watts \& Strogatz, 
11998 and Lazer \& Friedman, 2007), so our focus is not on the results of this setup per $s e^{7}$. The results in the global scenarios are far less distinguishable between conditions, suggesting that

3 diversity-preference contributes most to global cohesion when networks are local. Instead, by 4 opening up potential ties from the entire network, we can compare the performance of networks 5 and their emergent structures from instances when potential ties are sourced locally versus when

6 ties can come the entire network. Specifically, we can see how much performance is attributed to 7 the emergent network structure and how much performance benefits from exposure to diversity in 8 the collective problem-solving process, which we will explore in more depth later in the paper. memoryless: only the current performance of the agent is considered and not the rolling average

11 of its performance to date in the simulation. We find that our results remain consistent when agents

12 are endowed with memory. Agents will not search for new ties in a round if all their ties satisfy

13 the tie-searching criterion of its network. For agents in diversity-seeking networks, agents will not

14 look for new ties once all their ties are to differently typed agents. For merit-seeking networks, 15 agents will not look for new ties if all their ties are to agents whose score is at least as high as 16 theirs.

\section{4.1. Performance across the Three Network Setups}

20 We have three treatment conditions defined as the tie-search proclivities of agents in a network21 diversity-seeking networks, homophily-seeking networks, and merit-seeking networks—and four 22 different search heuristics that differentiate A and B species agents, defined in the Supplemental 23 Materials-Section A. For each treatment condition (e.g., diversity-seeking, homophily-seeking,

\footnotetext{
${ }^{7}$ In the Supplemental Materials-Section C, however, we loosen the local criterion and allow the potential pool of ties to come from the entire global network.
} 
1 merit-seeking), we run 10,000 simulations using 10,000 unique NK spaces. We compare the results

2 both in the aggregate, averaged by the treatment conditions across all NK spaces, and compare the 3 results among the three conditions between each NK space. 
A

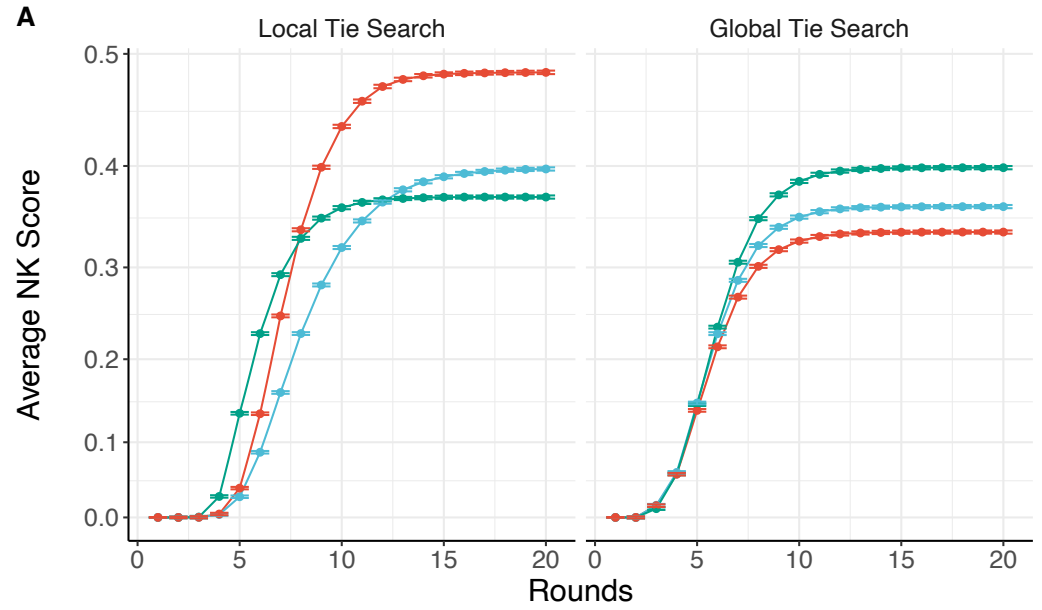

B
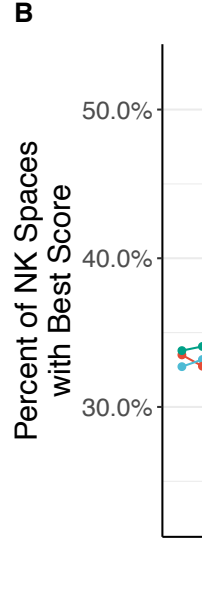

$\begin{array}{ll}\text { Homophily } & \rightarrow \text { Merit } \\ \text { Seeking } & \text { Seeking }\end{array}$

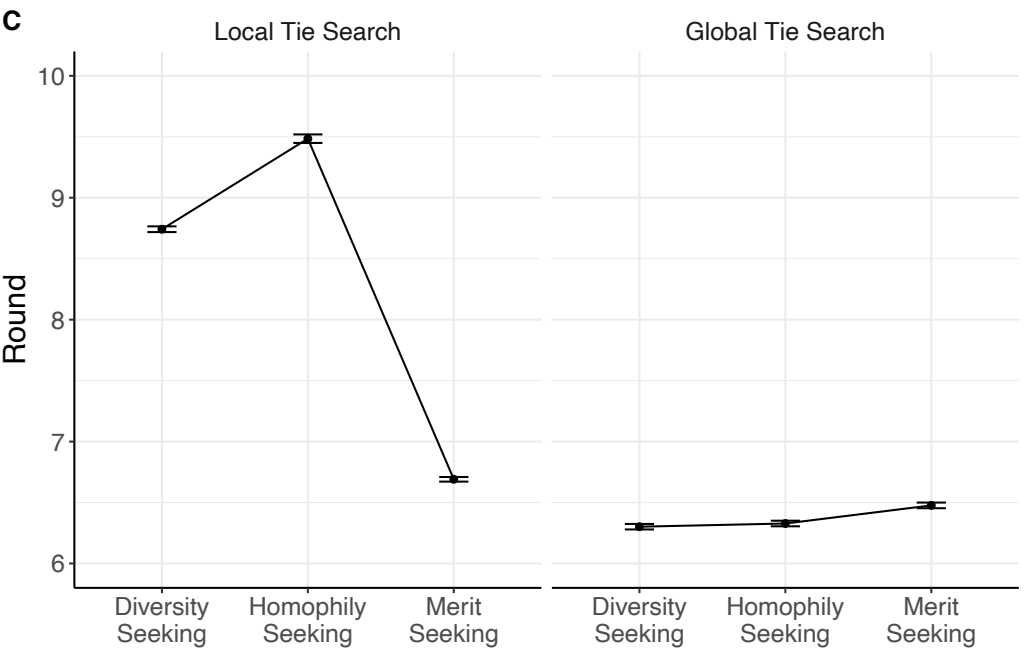

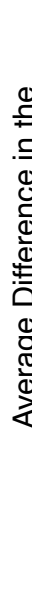

Local Tie Search

Global Tie Search

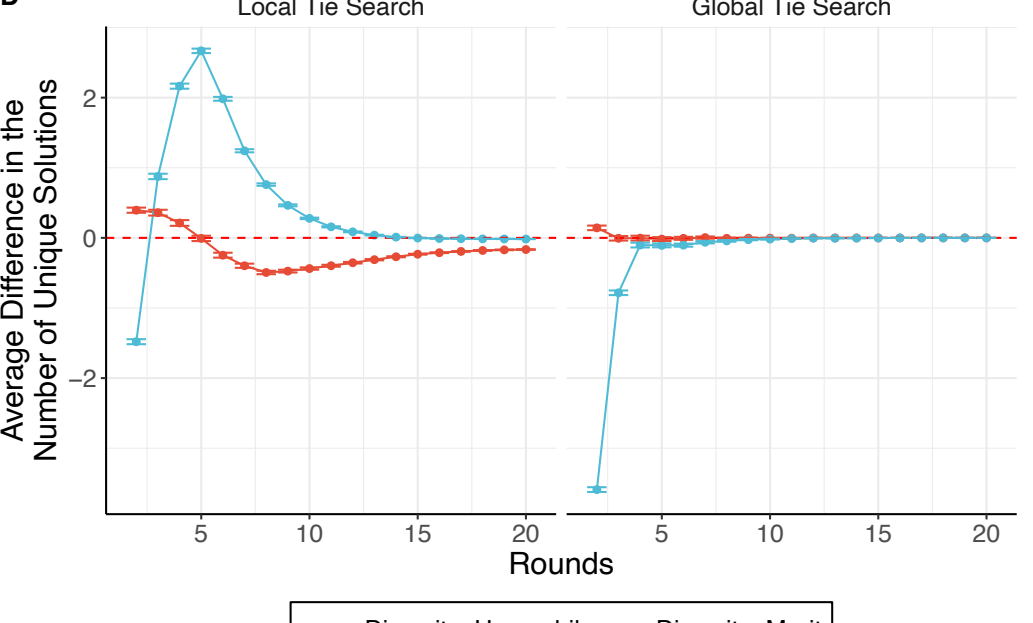

Figure 2. (A) Averaged normalized NK scores over time for the three treatment conditions: diversity-seeking networks, homophilyseeking networks, and merit-seeking networks. (B) The percentage of NK spaces that each network condition was performing the best as compared to the other conditions over time. (C) The average round where each network setup achieved equilibrium (D) The average difference in the number of unique solutions introduced into the network over time between diversity-seeking and merit-seeking networks in each round. 
In Figure 2, we plot several network metrics from our experiments. The panes on the left are for the network simulations where tie search is localized, the panes on the right are for when

3 tie search is global. We first plot the average score across all NK spaces for each of the three 4 treatments over time in Figure 2A. The $y$-axis is the normalized NK score ${ }^{8}$ and the $x$-axis indicates 5 the number of rounds. We include standard errors for these trends, as well. As shown in Figure 6 2A-local (left pane), while merit-seeking networks quickly settle to equilibrium and perform best

7 in the short-run, diversity-seeking networks find the highest solutions in the long-run and begin to 8 outperform merit-seeking networks on average by round 8 . Homophily-seeking networks also 9 begin to outperform merit-seeking networks on average by round 12, although at no point fare 10 better than diversity-seeking networks. The average NK score, however, masks performance 11 trends among the three network setups.

We now replot the trends in Figure 2B-local (left pane) to unpack these results. Instead of

13 plotting the average score across all NK spaces, we compare performance within each NK space.

14 Performance is defined at the average NK score of agents in the network at each round. For each

15 NK space, we count which of the three treatment conditions has the best score in each round on 16 the $y$-axis. For example, in round 5 we can see that the scores in about $52 \%$ of all NK spaces are 17 led by the merit-seeking condition. We also allow for ties, so the total percentage in each round 18 may sum to over $100 \%$. Here, the disparity in performance is much starker: Merit-seeking networks by far outperform diversity-seeking and homophily-seeking networks, in the short-run.

\footnotetext{
${ }^{8}$ We transform the NK scores to highlight the difference in performance among diversity-seeking, homophily-seeking, and merit-seeking networks. As the results are statistically different, this decision is meant to better visualize the difference in performance among the three network setups. Specifically, every NK score $x$ is transformed using the following scaling: $\left(\frac{x^{8}}{1-x^{8}}\right)^{2}$. This transform is just one of many possible techniques, but we choose it here to visually compress early round values that ascend quickly and separate later round values that distinguish the performance among the three networks.
} 
1 However, diversity-seeking networks find the best solution in the long-run, outperforming both 2 networks.

Figures 2A-local and 2B-local (left panes) together offer a complementary understanding

4 of how individual-seeking preferences shape systemic outcomes: Diversity-seeking networks

5 perform better in the long-run but merit-seeking networks do better in the short-run. Homophily-

6 seeking networks, by contrast, never provide the best solutions. However, each of these

7 visualizations offers a slightly different picture: Diversity-seeking networks appear far more

8 dominant in the long-run when looking at average score (Figure 2A-local) than the percentage of

9 best performances (Figure 2B-local) while merit-seeking networks appear much stronger in the

10 short-run when considering percentage of best performance (Figure 2B-local) than average score

11 (Figure 2A-local). To unpack this, we now examine the quality of winning solutions offered by

12 each heuristic to better understand these contrasts.

13

\section{4.2. Differences in the Solution Generating Process}

15 The success of diversity-seeking networks can be attributed to two mediating factors: Populations 16 with the diversity seeking heuristic introduce more exploration and the network structures they

17 create are both egalitarian and integrated. As a result, they take longer to settle into stable networks 18 of interaction and generate a broader array of potential solutions.

19 First, in Figure 2C-local, we show the average number of rounds it took each network setup 20 (on the $x$-axis) to achieve equilibrium — or when only one unique solution existed in the network-

21 along with its standard errors on the $y$-axis. Diversity-seeking and homophily-seeking networks 22 take the longest to achieve equilibrium (i.e., achieved around round 8.75 and 9.5, respectively), 23 while merit-seeking networks find equilibrium much earlier (i.e., around 6.5 rounds). 
In Figure 2D-local, we then plot the average difference in the number of unique solutions produced by diversity-seeking and the number of unique solutions produced by homophily-seeking

3 networks (Diversity - Homophily) and the number of unique solutions produced by merit-seeking

4 networks (Diversity - Merit) in each round, along with their standard-errors, on the $y$-axis. A

5 unique solution is defined in a similar manner to Lazer and Friedman (2007), where a unique

6 solution is defined as any NK solution that has never existed before in the simulation, where we

7 end the simulation once the number of unique solutions is one. On average, diversity-seeking

8 networks consistently introduce more unique solutions over time than merit-seeking networks,

9 particularly in the earlier rounds. By contrast, after the first few rounds diversity-seeking networks

10 introduce fewer unique solutions than homophily-seeking networks, as shown in Figure 2D-local.

11 However, homophily-seeking networks take the longest to achieve equilibrium, generally

12 introduce the fewest number of unique solutions, and still perform the worst among the three setups

13 at all timescales.

14 Taken together, diversity-seeking networks more commonly find better solutions in the

15 long-run. Many argue that more unique solutions open up more opportunities for better solutions

16 (Gomez \& Lazer, 2019; Lazer \& Friedman, 2007; Shore et al., 2015). And with more unique

17 solutions introduced, settling on a solution and thus achieving equilibrium takes longer. However,

18 we find that while merit-seeking networks introduce far fewer unique solutions than diversity-

19 seeking networks, homophily-seeking networks that take the longest to achieve equilibrium

20 introduce the most unique solutions. To better contextualize this in terms of the value of diversity,

21 we turn to analyzing the network structure across these three setups. 
In Figure 2A-global (right pane), we see that the results are much lower for global tie search networks, but the ordering among the three setups has changed. Now, merit-seeking performs the

3 best and diversity-seeking the worst.

In Figure 2B-global, again see the opposite finding: merit-seeking networks perform worse slightly better than homophily-seeking and diversity-seeking that mirrors the results in Figure 2A7 global.

In Figure 2C-global, we re-plot for the global network how many rounds it takes for the simulations on average to reach equilibrium on the $y$-axis for each type of network on the $x$-axis.

10 Again, we find the opposite result: while merit-seeking networks takes slightly longer to reach 11 equilibrium, but homophily-seeking and diversity-seeking networks are statistically tied.

In Figure 2D-global, we re-plot the number of unique solutions introduced for the global

13 tie search networks, finding a much more muted differential than in the localized networks and 14 little discernable difference between the trends. Merit-seeking networks introduce a few more 15 unique solutions earlier on into the network. We again plot the average difference in the number 16 of unique solutions produced by diversity-seeking and the number of unique solutions produced 17 by homophily-seeking networks (Diversity - Homophily) and the number of unique solutions 18 produced by merit-seeking networks (Diversity - Merit) in each round, along with their standard19 errors, on the $y$-axis. Again, we now do this for global tie search networks. On average, while 20 merit-seeking networks introduce three more unique solutions very early on in the simulation than 21 diversity-seeking networks, the total number of unique solutions introduced is relatively the same 22 across all three setups. For instance, homophily-seeking networks introduce nearly the same 23 number of unique solutions as diversity-seeking networks. 

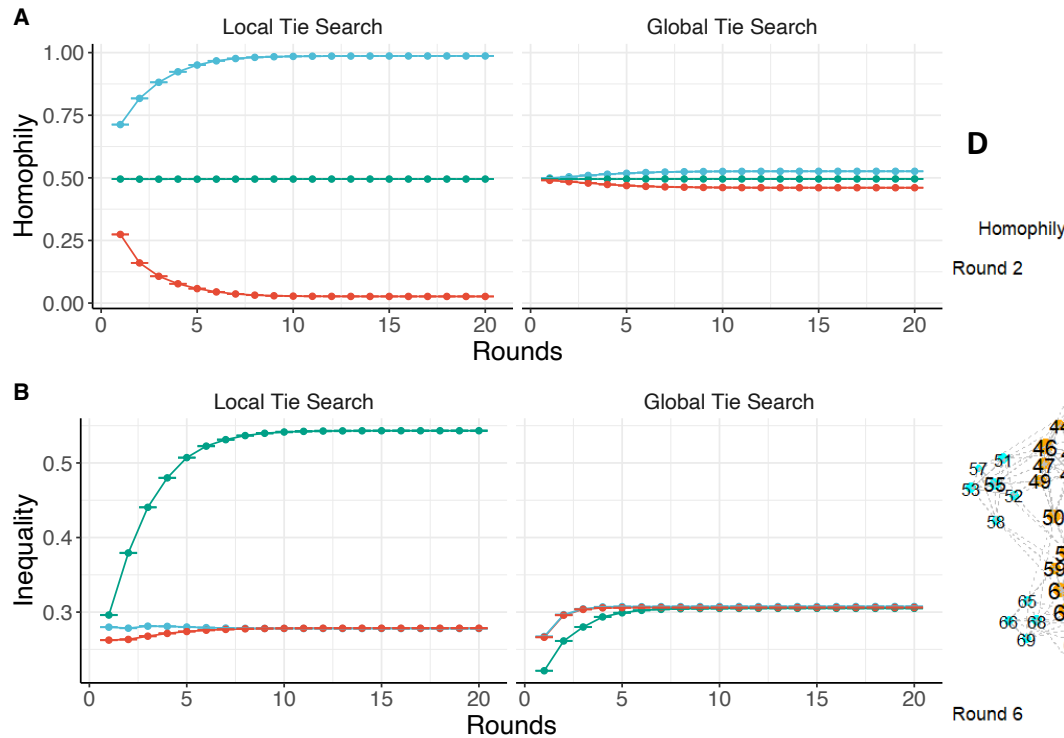

C Local Tie Search

Global Tie Search

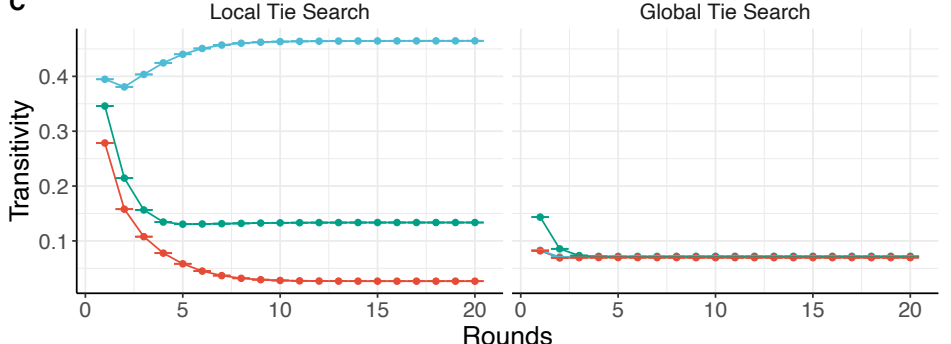

$\rightarrow \begin{aligned} & \text { Diversity } \\ & \text { Seeking }\end{aligned} \rightarrow \begin{aligned} & \text { Homophily } \\ & \text { Seeking }\end{aligned} \rightarrow$ Merit

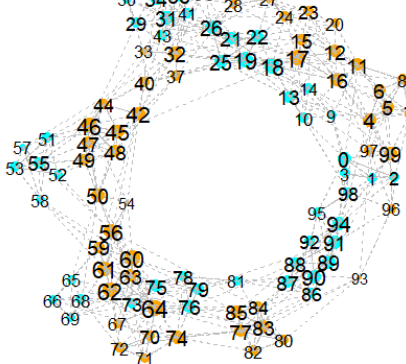

Round 6

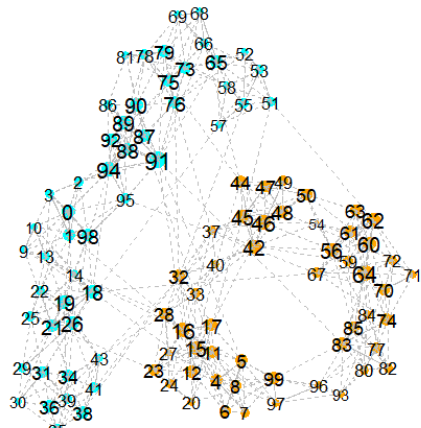

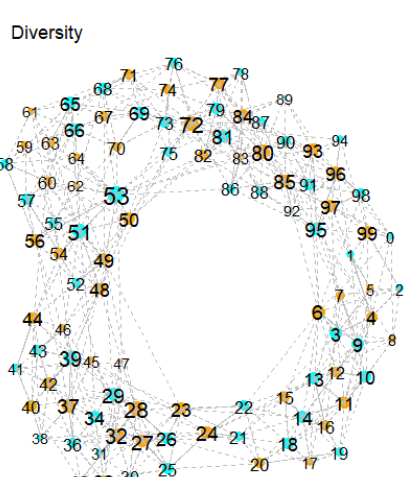

$353330-2$

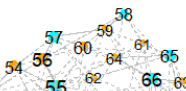

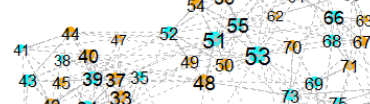

${ }_{46} 42 \quad 3429$

30,32
$28 \quad 25$
$28 \quad 26$

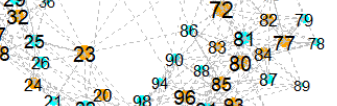
$24 \quad 22^{20} \quad 98^{94} 96_{91}^{85} \quad 87$ $9915^{15} 15^{16}$ $\begin{array}{r}0.6 \\ 4 \\ 4\end{array} 5^{11}>12$
Merit

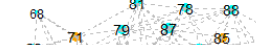
$74^{60} \quad 76 \quad 67 \quad 80 \quad 84^{86^{85}} 83 \quad 97$ $\begin{array}{lllllll}73 & 75 & 71 & 89 & 96 & 95 & 3\end{array}$ $64^{66} \quad 6354^{91} 8294 \quad 0 \quad 4$ $58 \quad 55 \quad 59 \quad 93 \quad 92 \quad 6 \quad 5$

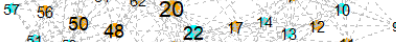
$49-52 \quad 25 \quad 19 \quad 15$ $45=\quad 47 \quad 23 \quad 26 \quad 26^{16} \quad$ is

44. $\quad \begin{array}{llll}39 & 38 & 28 & 28\end{array}$ $\begin{array}{llll}43 & 42 \quad 30 \quad 36 \quad 35 \\ 41 & 34\end{array}$

37. 31

87. 43 $\begin{array}{lllll}38 & 56 & 58 & 85 & 86\end{array}$ $19 \quad 6472 \quad 44 \quad 92 \quad \pi 2^{2}$ $7_{47}^{24} 55^{24} 90_{62^{89}}^{25} 94^{18}$

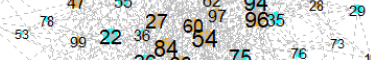
$40 . \quad 23 \cdot 12^{50} 16^{26} 0^{70}$

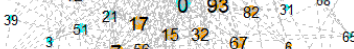
$45 \quad 3 \quad 756 \frac{15-32}{6} \quad 67 \quad 6$ 16. $4.91 \quad-41$

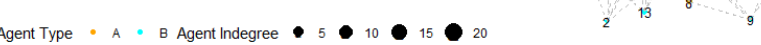

Figure 3. (A) Network homophily, the total proportion of ties between agents of the same type, for each of the three heuristics by round. (Round 1 reflects the network structure after the first round of tie-swaps, all network types are initialized with a random 'small-world' network and begin with an average homophily value of 0.5) (B) Network inequality, the Gini coefficient for the in-degree distribution for each of the three heuristics by round. (C) Network transitivity, the proportion of all possible triangles that exist in each network, for each of the three heuristics by round. (D) Example of a network's structural evolution. The state of each network at round 2 and round 6 for each of the three heuristics from the same sample NK-space. Node color indicates nodal type, and node size reflects nodal indegree. By round 6, homophily-seeking agents have formed two nearly separate clusters, diversity-seeking agents arrange themselves into a triangle-free and integrated torus structure, and merit-seeking agents have formed a core-periphery network. 


\section{4.3. Emergent Network Structures and Performance}

2 Individual preferences for diversity-seeking, homophily-seeking, or merit-seeking networks will

3 also influence the shape of the collaboration network. Figure 3 show how the networks' structural

4 metrics - homophily, inequality, and transitivity plotted on the $y$-axis - change over the course of

5 the simulations. Standard errors are included but are very small. Like the setup in Figure 2, the left

6 column panes are for when the tie search is localized, while the right column panes are simulations

7 for when the tie search is global. (In the Supplemental Materials-Section D, we show these network

8 measures across different neighborhood criteria as well as different NK landscapes.) Homophily

9 is a node-based measure, where the degree of homophily is based on how many agents have similar

10 neighbors, whereas inequality and transitivity are based on the distribution and structure of links.

11 Aside from network homophily in Figure 3A-local, which will obviously be much lower

12 in the diversity-seeking condition, there are two primary facets of network structure of interest.

13 First, we are interested in the network's in-degree distribution. This is visualized in Figure 3B-

14 local. In the merit-seeking condition, we expect that the inequality of this distribution (as

15 calculated with a Gini coefficient) will be much higher, as a small number of high-performing

16 agents should receive most directed ties. Second, we are interested in transitivity (the tendency for

17 triangles to form) within networks. This is shown in Figure 3C-local. We expect this will be lower

18 in the diversity-seeking condition, as any complete triad requires at least one connection between

19 agents of the same species.

20 Each of the three affiliation heuristics are associated with a different combination of

21 structural attributes. Homophily is (not surprisingly) highest for graphs of homophily-seeking

22 agents and lowest for diversity-seeking agents. Merit-seeking networks show complete

23 indifference to agent type, with ties split evenly between in-group and out-group. Inequality is

24 highest in merit-seeking networks, where a small number of early succeeders attract a great deal 
1 of attention. By contrast, diversity-seeking and homophily-seeking networks are highly

2 egalitarian, with homophily-seeking networks having slightly higher levels of inequality in the

3 opening rounds compared to diversity-seeking networks, and more consistent values of inequality.

4 Transitivity is the percentage of all possible triads (or triangles, defined as two links with at least

5 one shared node) in a network ${ }^{9}$. Transitivity reveals the presence of tightly interconnected

6 communities. In homophily-seeking networks, high rates of in-group interaction (reflected in nodal

7 homophily) lead to high levels of graph clustering and triangle formation. Whereas both

8 homophily and transitivity are at an absolute minimum for diversity-seeking networks ${ }^{10}$, and are

9 at middling levels for merit-seeking networks.

The emergent network structures for diversity-seeking, homophily-seeking, and merit-

11 seeking networks are similar for the global tie search condition (right panes). For homophily

12 (Figure 3A-global), all three networks are qualitatively similar to one another, with little

13 substantive difference in their degrees of homophily when compared to the more dramatic

14 differences in the localized setup. For inequality and transitivity, the emergent networks in the

15 long-run are, on average, similar in how unequal their in-degree ties are and their degree of

16 clustering. Indeed, when we plot sample networks in Figure 3B-global and 3C-global, we see that

17 the network structures are fairly similar across the three setups.

18 To better illustrate how network structure unfolds within individual simulations, Figure 3D

19 shows the evolution of sample diversity-seeking, merit-seeking, and homophily-seeking networks

20 after 1 timestep and 5 timesteps (i.e., $2^{\text {nd }}$ and $6^{\text {th }}$ round, respectively). Figure 3D is for networks

21 with a localized tie search, as the global tie search networks have no discernable structure as shown

\footnotetext{
${ }^{9}$ This was calculated using the Python module Networkx and its cluster transitivity algorithm.

${ }^{10}$ On the other hand, it is impossible for a completely diverse graph with two-agent types to have any triangles from a graph-theoretic perspective.
} 
1 in Figures 3A-C. All networks begin from a torus, where each agent (numbered from 0 through

299 ) is connected to the three agents on either side numerically (e.g., agent 2 is connected to 99,0 ,

$31,3,4$, and 5). We see that the evolution of the diversity-seeking graph from the initial torus shows

4 the potential structural advantages of this approach. Unlike merit-seeking networks, innovation is

5 not dominated and controlled by a handful of early succeeders. Furthermore, the graph does not

6 have large, isolated clusters between agent types, which can be seen in the homophily-seeking

7 network. What evolves is a highly decentralized network that is still immune from local clustering.

8 Each individual preference leads to a distinct network structure, and the one created by diversity9 seeking preferences is well-suited to generate and disseminate high-quality solutions.

\section{4.4. Why Diversity Works: Structure and Perspective}

12 We posit that the long-term success of diversity-seeking networks is in part due to an emergent

13 structure that allows solutions to slowly reach farther reaches of the network, and in part to because

14 diversity-seeking behavior engenders higher quality solutions. Consistent with past work, we find 15 that homophily-seeking networks introduced the largest number of unique solutions. This is 16 because the emergent network structure fostered by homophily-seeking behavior takes longer to 17 reach equilibrium than merit-seeking networks, allowing for more ideas to spread across in the 18 network. However, we find that the quality of these solutions is on average much worse than 19 diversity-seeking networks, even though these networks take longer to reach equilibrium and 20 introduce more unique solutions. This contrasts with merit-seeking networks that are much more 21 fully interconnected, albeit through a handful of initially higher performing agents. 


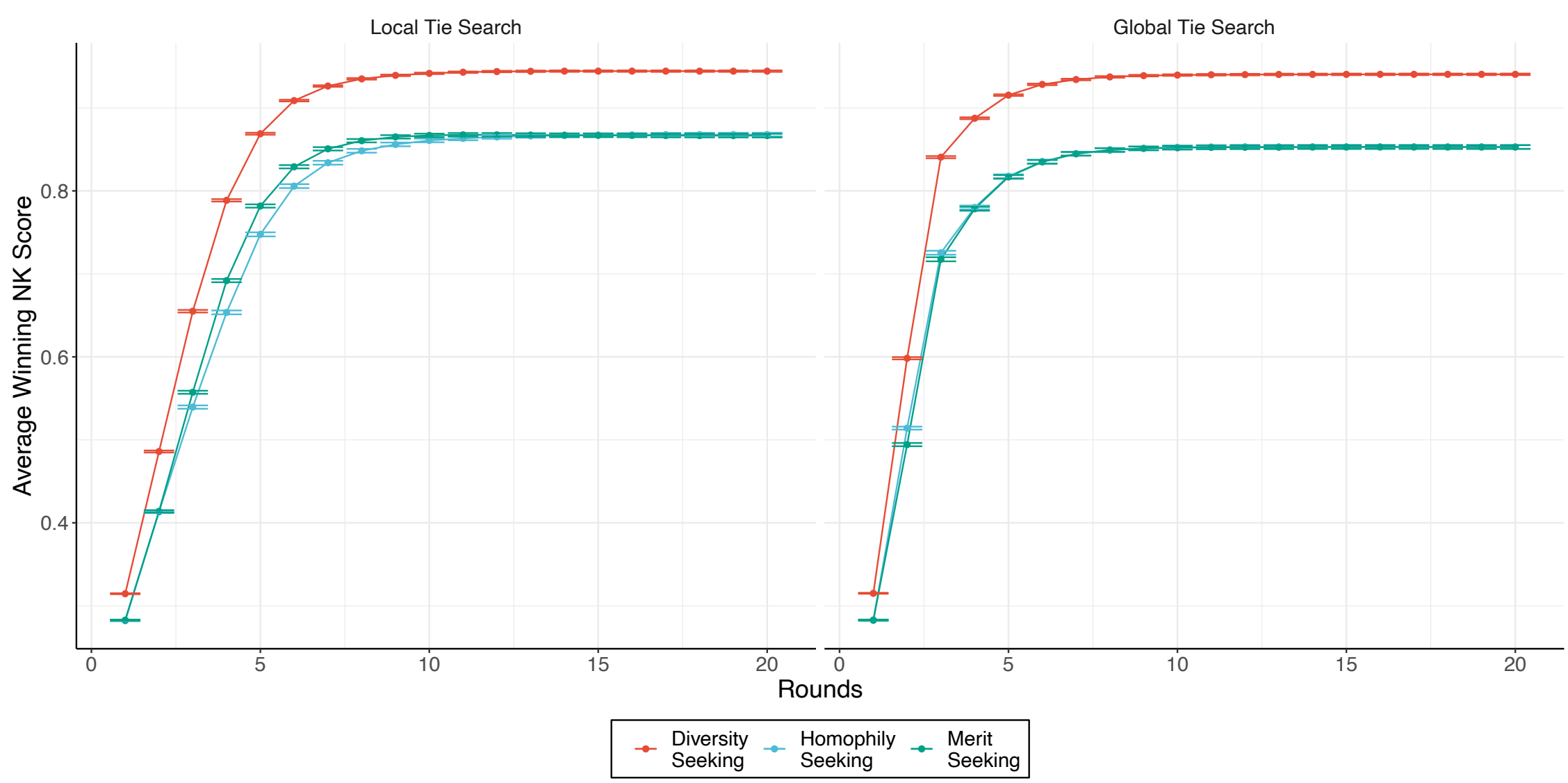

2 Figure 4. The average NK score of networks that find the best score among the three setups over time. 
Figure 4 is comparable to Figure $2 \mathrm{~A}$ but only includes the average NK score of the

2 simulations that "won" each NK space on the $y$-axis. Each NK space is only represented in Figure

34 by one of the three setups. For example, if a merit-seeking network in a given NK space found

4 the best score as compared to diversity-seeking or homophily-seeking at equilibrium, we count the

5 performance in this NK space for merit-seeking's average NK score (and similarly for the other

6 setups). While we exclude ties from these calculations, ties constituted around $0.05 \%$ of all NK

7 space results. From these "best performances" for each of the three conditions, we take their

8 average NK scores over time, along with their respective standard errors. When diversity-seeking

9 networks offer the best solution, the score tends to be much higher. In other words, the quality of

10 the solutions that diversity-seeking networks find is consistently better than homophily-seeking or

11 merit-seeking networks. This holds across all time scales. Diversity-seeking networks offer higher

12 levels of variance in performance. These networks tend to outperform their merit-seeking and

13 homophily-seeking networks counterparts more frequently; and when they do, diversity-seeking

14 networks tend to win "big": diversity-seeking networks outperform their counterparts by a much

15 greater extent than their counterparts.

We find that diversity-seeking networks consistently find the highest NK scores on average

17 when they win. In other words, the quality of the diversity-seeking networks, even with a different

18 network structure that is consistent across the three setups and different than in the localized tie

19 results, remains consistently higher. Taken together, we compare how diversity improves systemic

20 performance based on how the network structure allows for solutions to dissipate through the

21 network. We find that diversity leads to the generation of higher quality solutions and, when given

22 enough time, diversity-seeking allows for these solutions the opportunity to disseminate to the rest

23 of the network. In both global and localized tie search conditions, diversity-seeking networks find 
1 the highest quality solutions among the setups when they perform the best. Our focus here is not

2 whether information should be localized or made more global per se, as prior work has explored

3 the implications of global and local access to knowledge that our findings corroborate here (again 4 see Watts \& Strogatz, 1998 and Lazer \& Friedman, 2007).

\section{5. Discussion}

7 We find that diversity-seeking agents do well in collective-problem solving networks because

8 interactions between diverse agents not only generate higher quality solutions, but the resulting

9 network structure of diversity-seeking agents also better facilitates the diffusion of solutions.

10 Whom we seek information from is highly driven by individual preferences that can affect

11 systemic outcomes. Building upon the extensive tradition of agent-based modelling (ABM) of

12 collective-solving networks, we develop communicative torus networks of diverse problem-

13 solving agents tasked with solving a complex problem represented by Kauffman's NK space.

14 Agents exploit their neighbors' solutions or explore for a new one using their unique and diverse

15 way with which they manipulate their NK solution. While our model is founded on the well-

16 established "exploration and exploitation" model of collective problem solving (De Marchi \&

17 Page, 2014; March, 1991), our unique contribution and extension is the ability of agents to see out

18 new ties among their local neighborhoods in the network.

Our finding offers insights to previously overlooked consequences of diversity by bridging

20 the well-established literature on the benefits of exposure to diversity in problem solving with the

21 literature on the consequences of endogenous communicative structures. For instance, previous

22 work suggests that network structure and exposure to diversity leads to more opportunities for

23 exploration and thus a greater probability of finding higher quality solutions (Boroomand \&

24 Smaldino, 2021; Gomez \& Lazer, 2019; Lazer \& Friedman, 2007). We instead find that while 
1 network structure does foster the introduction of more unique solutions since it inhibits the flow

2 of information, the success of these setups is predicated on the quality of the solutions that uniquely

3 come back from exposure to diversity rather than the sheer number of solutions introduced. We

4 ultimately find that a truly integrative diversity, where individual actors do not only nominally co-

5 exist, but pro-actively seek out one another's unique skills and perspectives, offers more promising

6 results. This result aligns with empirical finding that diversity is more beneficial for organizations

7 pursuing more innovative strategies (Richard et al., 2003). However, building a diverse network

8 and generating solutions that incorporate different abilities and perspectives requires time and

9 patience. The framework provided by our approach not only builds upon a classic framing within

10 the organizational science and decision-making literatures, but also allows us to extend our

11 findings to scholars in a variety of fields.

12 The most direct application is widening our standing of which diversity-based practices

13 could lead to better collective outcomes when individual problem-solvers (e.g., researchers,

14 scientists, engineers, policymakers, etc.) are given agency in choosing their collaborative

15 arrangements. We see that while emulating successful behaviors may work at first, better solutions

16 may come from paying attention to people who have a different set of skills or perspectives.

17 However, in situations where timeliness is important, integrating diverse perspectives may not

18 provide the same rewards.

19 Those interested in science, technology, innovation, or creative industries may be less

20 concerned with locating optimal solutions, and more interested in the variety of answers provided

21 through collaboration. Diversity-seeking populations again out-perform merit-seeking populations

22 in this regard, as populations that emulate success will naturally converge on a small subset of

23 solutions. However, homophily-seeking populations outperform diversity-seeking populations 
1 here. Separate clusters of individuals can generate separate sets of solutions since solutions will

2 not cross-contaminate. However, this is due to the network structure induced by homophily, and

3 not homophily itself. Prior work has shown the benefits of isolated subgroups in organizational

4 settings (Fang et al., 2010). Clustering within academic disciplines, for example, may help

5 maintain diversity of thought within the scientific community. However, clustering along

6 something more orthogonal to the structure of knowledge (i.e., geographic region, language) may

7 also create a more integrated form of intellectual diversity.

\section{5.1. Limitations and Extensions}

10 Our model lays the foundation for future extensions that can build upon several of its limitations.

11 For instance, merit-seeking networks are memoryless systems: How "merit" is assessed is based

12 on the current performance of agents, as opposed to their cumulative performance over multiple

13 rounds. While we tested a memory-based form of "merit" in a limited capacity and found that our

14 results are qualitatively similar, expanding on how "merit" is conceptualized and perceived by 15 others warrants further exploration.

16 Diversity, as we have set it up, assumes no cost to communication. In many settings, 17 communicative costs are part-and-parcel with diversity. This can take the form of being "lost in 18 translation" (e.g., physicists and sociologists struggling to understand one another), or a cost 19 associated with a differing agent (e.g., a price for an external consultant). Mistranslations or errors

20 in copying have been shown to enhance systemic performance, as these moments are serendipitous

21 and open new pathways to better solutions. This is often the crux of scientific and innovative 22 discoveries. There can also be a penalty associated with working with others. Prejudice, biases, 23 and social stigma are commonplace. As such, diverse collaborations may carry a penalty or tax 
1 associated with scores. Such an extension of this model may reveal that the benefits of diverse

2 collaborations as outlined here may carry much greater costs.

A large benefit to diversity is not just exposure, but how people learn how from others, as well. In our model, diversity is preordained and static. However, along with "exploiting" solutions

5 from other agents, agents may also exploit the heuristics of other agents, as well. Alternatively, 6 agents can wholesale copy the heuristic of a species of an agent who has a better solution. 7 Extensions of our model that allow for entire or partial copying of heuristics, or even unintended 8 mutations, opens new venues for research on diversity in collective problem solving.

Our model of diversity rests on the premise that diversity is complementary and salient to

10 the problem at hand. Little work has explored how complementarity or salience shapes diversity's 11 efficacy in collective problem solving. Gomez and Lazer (2019) showed how complementary and 12 nested forms of diversity are both beneficial, but where nested forms of diversity lead to even 13 better outcomes. match empirical settings and its data that validates our findings. To be sure, ABMs are valid in their own right, as the rich tradition of "exploration and exploitation" work demonstrates.

17 Nonetheless, further validating our results enhances the verisimilitude of ABMs. For instance, 18 Shore, et al. (2015) use focus groups to validate many findings of "exploration and exploitation" 19 ABMs.

20 Finally, future work may also benefit from simultaneously considering different types of 21 diversity, mainly task-relevant and task-irrelevant diversity. The integration or segregation of 22 individuals along the lines of task-irrelevant diversity may change the relationship between 23 diversity-seeking agents and solution success. Agents who are homophily-seeking along the lines 
1 of task-irrelevant traits could create separate clusters of individuals who are heterogeneous along

2 task-relevant attributes, combining the creative benefits of clustered networks without sacrificing

3 an integration of diverse perspectives. While this would cause obvious harms if task-irrelevant

4 homophily reflected traditional fault lines of marginalization, encouraging segregation along the

5 lines of trivial and artificially induced categories could be a promising strategy for organizational

6 success. Indeed, many universities and high schools have experimented with this by soring their

7 students into "houses" or "colleges" that are internally heterogeneous (see Nelson, Johnson, and

8 Boes 2012 for a description of one such system) but provide a potential axis for homophily and 9 clustering that is largely orthogonal to the broader inequities of society.

\section{6. Conclusion}

12 We show that collectives where agents seek out diversity are highly successful because they both

13 promote more exploration that yields higher quality solutions and foster network structures that

14 better disseminate these solutions. Ultimately, our model decomposes why agent-driven diversity

15 may be an important element for organizations and collectives seeking to capitalize on their diverse

16 composition. The model of integrative problem-solving presented here helps shift our

17 understanding of diversity in problem-solving networks from one of distribution to one of

18 integration, and simultaneously accounts for the importance of agency and structure within

19 collaborative processes. Our study offers a valuable starting point for tests of more sophisticated

20 models of collaboration going forward. Empirical and experimental research has examined the

21 challenges of creating school and work environments that values both diversity and merit

22 (Gündemir et al., 2017; Walton et al., 2013). Future models may capture more theoretical insight

23 by examining both preferences simultaneously, incorporating more cognitively sophisticated 
1 agents that create or respond to stereotypes, or adding communication challenges when

2 information is shared between different types of agents.

\section{7. References}

5 Aggarwal, I., \& Woolley, A. W. (2019). Team creativity, cognition, and cognitive style diversity. Management Science, 65(4), 1586-1599.

Aral, S., \& Van Alstyne, M. (2011). The Diversity-Bandwidth Trade-off. American Journal of Sociology, 117(1), 90-171. https://doi.org/10.1086/661238

Arentze, T. A., Kowald, M., \& Axhausen, K. W. (2013). An agent-based random-utilitymaximization model to generate social networks with transitivity in geographic space. Social Networks, 35(3), 451-459.

Banisch, S., Lima, R., \& Araújo, T. (2012). Agent based models and opinion dynamics as Markov chains. Social Networks, 34(4), 549-561.

Becker, J., Brackbill, D., \& Centola, D. (2017). Network dynamics of social influence in the wisdom of crowds. Proceedings of the National Academy of Sciences, 114(26), E5070E5076. https://doi.org/10.1073/pnas.1615978114

Boone, C., \& Hendriks, W. (2009). Top management team diversity and firm performance: Moderators of functional-background and locus-of-control diversity. Management Science, 55(2), 165-180.

Boroomand, A., \& Smaldino, P. E. (2021). Hard Work, Risk-Taking, and Diversity in a Model of Collective Problem Solving. Journal of Artificial Societies and Social Simulation, 24(4).

Burt, R. S. (1995). Structural Holes. Harvard University Press.

Burt, R. S. (2004). Structural Holes and Good Ideas. American Journal of Sociology, 110(2), 349-399. https://doi.org/10.1086/421787 
1 Campbell, L. G., Mehtani, S., Dozier, M. E., \& Rinehart, J. (2013). Gender-heterogeneous working groups produce higher quality science. PloS One, 8(10), e79147.

3 Castilla, E. J. (2008). Gender, Race, and Meritocracy in Organizational Careers. American Journal of Sociology, 113(6), 1479-1526. https://doi.org/10.1086/588738

Castilla, E. J., \& Benard, S. (2010). The Paradox of Meritocracy in Organizations. Administrative Science Quarterly, 55(4), 543-576.

Chancellor, J., Layous, K., Margolis, S., \& Lyubomirsky, S. (2017). Clustering by well-being in workplace social networks: Homophily and social contagion. Emotion, 17(8), 11661180. https://doi.org/10.1037/emo0000311

Chang, M.-H., \& Harrington Jr, J. E. (2006). Agent-based models of organizations. Handbook of Computational Economics, 2, 1273-1337.

Csaszar, F. A. (2018). A note on how NK landscapes work. Journal of Organization Design, $7(1), 1-6$.

Cummings, J. N. (2004). Work groups, structural diversity, and knowledge sharing in a global organization. Management Science, 50(3), 352-364.

De Marchi, S., \& Page, S. E. (2014). Agent-based models. Annual Review of Political Science, $17,1-20$.

de Oliveira Maciel, C., \& Netto, R. Z. R. (2020). Architectural agency in intra-organizational networks. Journal of Business Research, 109, 489-497. https://doi.org/10.1016/j.jbusres.2018.12.006

DellaPosta, D. (2020). Pluralistic collapse: The "oil spill" model of mass opinion polarization. American Sociological Review, 85(3), 507-536. 
1 den Hamer, P., \& Frenken, K. (2020). A network-based model of exploration and exploitation. Journal of Business Research.

3 Derex, M., Bonnefon, J.-F., Boyd, R., \& Mesoudi, A. (2019). Causal understanding is not necessary for the improvement of culturally evolving technology. Nature Human Behaviour, 3(5), 446-452.

Epstein, J. M., \& Axtell, R. (1996). Growing artificial societies: Social science from the bottom up. Brookings Institution Press.

Fang, C., Lee, J., \& Schilling, M. A. (2010). Balancing exploration and exploitation through structural design: The isolation of subgroups and organizational learning. Organization Science, 21(3), 625-642.

Fiol, C. M. (1994). Consensus, diversity, and learning in organizations. Organization Science, $5(3), 403-420$.

Fioretti, G. (2013). Agent-based simulation models in organization science. Organizational Research Methods, 16(2), 227-242.

Fontanari, J. F., \& Rodrigues, F. A. (2016). Influence of network topology on cooperative problem-solving systems. Theory in Biosciences, 135(3), 101-110. https://doi.org/10.1007/s12064-015-0219-1

Goldberg, A., \& Stein, S. K. (2018). Beyond social contagion: Associative diffusion and the emergence of cultural variation. American Sociological Review, 83(5), 897-932.

Gomez, C. J., \& Lazer, D. M. J. (2019). Clustering knowledge and dispersing abilities enhances collective problem solving in a network. Nature Communications, 10(1), 1-11. https://doi.org/10.1038/s41467-019-12650-3 
1 Granovetter, M. S. (1973). The Strength of Weak Ties. American Journal of Sociology, 78(6), $1360-1380$.

3 Gündemir, S., Dovidio, J. F., Homan, A. C., \& De Dreu, C. K. (2017). The impact of organizational diversity policies on minority employees' leadership self-perceptions and goals. Journal of Leadership \& Organizational Studies, 24(2), 172-188.

Harrison, D. A., Price, K. H., Gavin, J. H., \& Florey, A. T. (2002). Time, teams, and task performance: Changing effects of surface-and deep-level diversity on group functioning. Academy of Management Journal, 45(5), 1029-1045.

Henry, A. D., Pralat, P., \& Zhang, C.-Q. (2011). Emergence of segregation in evolving social networks. Proceedings of the National Academy of Sciences, 108(21), 8605-8610. https://doi.org/10.1073/pnas.1014486108

Hong, L., \& Page, S. E. (2001). Problem Solving by Heterogeneous Agents. Journal of Economic Theory, 97(1), 123-163. https://doi.org/10.1006/jeth.2000.2709

Hong, L., \& Page, S. E. (2004). Groups of diverse problem solvers can outperform groups of high-ability problem solvers. Proceedings of the National Academy of Sciences of the United States of America, 101(46), 16385-16389. https://doi.org/10.1073/pnas.0403723101

Hoogendoorn, S., Oosterbeek, H., \& Van Praag, M. (2013). The impact of gender diversity on the performance of business teams: Evidence from a field experiment. Management Science, 59(7), 1514-1528.

Jackson, S. (1996). The consequences of diversity in multidisciplinary work teams. In Handbook of work group psychology (pp. 53-76). 
1 Kauffman, S. A. (1993). The origins of order: Self-organization and selection in evolution. Oxford University Press, USA.

3 Khraisha, T., \& Mantegna, R. N. (2020). Network structure and optimal technological innovation. Journal of Complex Networks, 8(1), cnz020.

Kleinbaum, A. M., Stuart, T. E., \& Tushman, M. L. (2013). Discretion Within Constraint: Homophily and Structure in a Formal Organization. Organization Science, 24(5), 13161336. https://doi.org/10.1287/orsc.1120.0804

Kollman, K., Miller, J. H., \& Page, S. E. (2000). Consequences of nonlinear preferences in a federal system. Political Complexity: Nonlinear Models of Politics, 23-45.

Lamm, A., Shoulders, C., Roberts, G., Irani, T., Snyder, L., \& Brendemuhl, J. (2012). The Influence of Cognitive Diversity on Group Problem Solving Strategy. Journal of Agricultural Education, 53(1), 18-30. https://doi.org/10.5032/jae.2012.01018

Lazer, D., \& Friedman, A. (2007). The Network Structure of Exploration and Exploitation. Administrative Science Quarterly, 52(4), 667-694. https://doi.org/10.2189/asqu.52.4.667

Leavitt, H. J. (1960). Task ordering and organizational development in the common target game. Behavioral Science, 5(3), 233-239.

Levinthal, D. A. (1997). Adaptation on Rugged Landscapes. Management Science, 43(7), 934950. https://doi.org/10.1287/mnsc.43.7.934

Lorenz, J., Rauhut, H., Schweitzer, F., \& Helbing, D. (2011). How social influence can undermine the wisdom of crowd effect. Proceedings of the National Academy of Sciences, 108(22), 9020-9025. https://doi.org/10.1073/pnas.1008636108 
1 Macy, M. W., \& Willer, R. (2002). From Factors to Actors: Computational Sociology and Agent-Based Modeling. Annual Review of Sociology, 28(1), 143-166. https://doi.org/10.1146/annurev.soc.28.110601.141117

Manzo, G., \& Baldassarri, D. (2015). Heuristics, Interactions, and Status Hierarchies: An Agentbased Model of Deference Exchange. Sociological Methods \& Research, 44(2), 329-387. https://doi.org/10.1177/0049124114544225

March, J. G. (1991). Exploration and Exploitation in Organizational Learning. Organization Science, 2(1), 71-87. https://doi.org/10.1287/orsc.2.1.71

March, J. G., \& Simon, H. A. (1993). Organizations (2 edition). Wiley-Blackwell.

Martins, L. L., Schilpzand, M. C., Kirkman, B. L., Ivanaj, S., \& Ivanaj, V. (2013). A contingency view of the effects of cognitive diversity on team performance: The moderating roles of team psychological safety and relationship conflict. Small Group Research, 44(2), 96126.

McLeod, P. L., Lobel, S. A., \& Cox Jr, T. H. (1996). Ethnic diversity and creativity in small groups. Small Group Research, 27(2), 248-264.

McPherson, J. M., \& Smith-Lovin, L. (1987). Homophily in Voluntary Organizations: Status Distance and the Composition of Face-to-Face Groups. American Sociological Review, 52(3), 370-379. https://doi.org/10.2307/2095356

McPherson, M., Smith-Lovin, L., \& Cook, J. M. (2001). Birds of a Feather: Homophily in Social Networks. Annual Review of Sociology, 27(1), 415-444.

https://doi.org/10.1146/annurev.soc.27.1.415 
1 Mello, A. L., \& Rentsch, J. R. (2015). Cognitive Diversity in Teams: A Multidisciplinary Review. Small Group Research, 46(6), 623-658. https://doi.org/10.1177/1046496415602558

Miller, J. H., \& Page, S. E. (2009). Complex adaptive systems: An introduction to computational models of social life. Princeton university press.

6 Milliken, F. J., \& Martins, L. L. (1996). Searching for common threads: Understanding the multiple effects of diversity in organizational groups. Academy of Management Review, 21(2), 402-433.

Mitchell, R., Nicholas, S., \& Boyle, B. (2009). The Role of Openness to Cognitive Diversity and Group Processes in Knowledge Creation. Small Group Research, 40(5), 535-554. https://doi.org/10.1177/1046496409338302

Nelson, S., Johnson, L., \& Boes, L. (2012). Harvard houses: The value of the tutorial system. About Campus, 17(2), 22-25.

Noë, N., Whitaker, R. M., Chorley, M. J., \& Pollet, T. V. (2016). Birds of a feather locate together? Foursquare checkins and personality homophily. Computers in Human Behavior, 58, 343-353. https://doi.org/10.1016/j.chb.2016.01.009

Olson, B. J., Parayitam, S., \& Bao, Y. (2007). Strategic Decision Making: The Effects of Cognitive Diversity, Conflict, and Trust on Decision Outcomes. Journal of Management, 33(2), 196-222. https://doi.org/10.1177/0149206306298657

Page, S. E. (2007). Making the Difference: Applying a Logic of Diversity. Academy of Management Perspectives, 21(4), 6-20. https://doi.org/10.5465/AMP.2007.27895335 
1 Page, S. E. (2008). The Difference: How the Power of Diversity Creates Better Groups, Firms, Schools, and Societies (New Edition). Princeton University Press. http://www.amazon.com/The-Difference-Diversity-Creates-Societies/dp/0691138540

Palley, A. B., \& Soll, J. B. (2019). Extracting the wisdom of crowds when information is shared. Management Science, 65(5), 2291-2309.

Putnam, R. D. (2000). Bowling alone: America's declining social capital. In Culture and politics (pp. 223-234). Springer.

Ren, Y., Chen, J., \& Riedl, J. (2016). The impact and evolution of group diversity in online open collaboration. Management Science, 62(6), 1668-1686.

Richard, O., McMillan, A., Chadwick, K., \& Dwyer, S. (2003). Employing an Innovation Strategy in Racially Diverse Workforces: Effects On Firm Performance. Group \& Organization Management, 28(1), 107-126. https://doi.org/10.1177/1059601102250022

Rivkin, J. W. (2000). Imitation of Complex Strategies. Management Science, 46(6), 824-844. https://doi.org/10.1287/mnsc.46.6.824.11940

Rivkin, J. W., \& Siggelkow, N. (2003). Balancing search and stability: Interdependencies among elements of organizational design. Management Science, 49(3), 290-311.

Rulke, D. L., \& Galaskiewicz, J. (2000). Distribution of knowledge, group network structure, and group performance. Management Science, 46(5), 612-625.

Schelling, T. C. (1969). Models of Segregation. American Economic Review, 59(2), 488-493. https://doi.org/10.2307/1823701

Shi, Y., Dokshin, F. A., Genkin, M., \& Brashears, M. E. (2017). A member saved is a member earned? The recruitment-retention trade-off and organizational strategies for membership growth. American Sociological Review, 82(2), 407-434. 
1 Shin, S. J., Kim, T.-Y., Lee, J.-Y., \& Bian, L. (2012). Cognitive team diversity and individual team member creativity: A cross-level interaction. Academy of Management Journal, 55(1), 197-212.

Shore, J., Bernstein, E., \& Lazer, D. (2015). Facts and figuring: An experimental investigation of network structure and performance in information and solution spaces. Organization Science, 26(5), 1432-1446.

Shugars, S. (2021). Good decisions or bad outcomes? A model for group deliberation on valueLaden topics. Communication Methods and Measures, 15(4), 273-291.

Siggelkow, N., \& Levinthal, D. A. (2003). Temporarily divide to conquer: Centralized, decentralized, and reintegrated organizational approaches to exploration and adaptation. Organization Science, 14(6), 650-669.

Siggelkow, N., \& Rivkin, J. W. (2009). Hiding the evidence of valid theories: How coupled search processes obscure performance differences among organizations. Administrative Science Quarterly, 54(4), 602-634.

Sirianni, A. D. (2018). Expectations and Coordination in Small Groups. In Advances in Group Processes (Vol. 35, pp. 181-207). Emerald Publishing Limited. https://doi.org/10.1108/S0882-614520180000035008

Skopek, J., Schulz, F., \& Blossfeld, H.-P. (2011). Who contacts whom? Educational homophily in online mate selection. European Sociological Review, 27(2), 180-195.

Smith, E. R., \& Conrey, F. R. (2007). Agent-based modeling: A new approach for theory building in social psychology. Personality and Social Psychology Review, 11(1), 87-104. 
1 Tegarden, D. P., Tegarden, L. F., \& Sheetz, S. D. (2009). Cognitive factions in a top management team: Surfacing and analyzing cognitive diversity using causal maps. Group Decision and Negotiation, 18(6), 537-566.

4 Tyre, M. J., \& Von Hippel, E. (1997). The situated nature of adaptive learning in organizations. Organization Science, 8(1), 71-83.

6 Uzzi, B., \& Spiro, J. (2005). Collaboration and Creativity: The Small World Problem. American Journal of Sociology, 111(2), 447-504. https://doi.org/10.1086/432782

8 Walton, G. M., Spencer, S. J., \& Erman, S. (2013). Affirmative Meritocracy. Social Issues and Policy Review, 7(1), 1-35. https://doi.org/10.1111/j.1751-2409.2012.01041.x

Watts, C., \& Gilbert, N. (2011). Does cumulative advantage affect collective learning in science?

An agent-based simulation. Scientometrics, 89(1), 437-463. https://doi.org/10.1007/s11192-011-0432-8

Watts, D. J., \& Strogatz, S. (1998). Collective dynamics of 'small-world'networks. Nature, 393(6684), 440-442.

15 Wegge, J., Roth, C., Neubach, B., Schmidt, K.-H., \& Kanfer, R. (2008). Age and gender 16 diversity as determinants of performance and health in a public organization: The role of task complexity and group size. Journal of Applied Psychology, 93(6), 1301. 


\section{Supplemental Materials}

\section{A. Agent Search Heuristics}

3 In our exploration and exploitation model, when agents cannot exploit the solutions of their neighbors, they

4 will move on to explore the NK problem space. To ensure that our results are not driven by any one 5 particular set of heuristics, we design four different types of heuristics. Two heuristics are randomly 6 assigned to an NK space at the start of each simulation. As such, referring to an "A" species agent or a "B" 7 species agents does not reference any particular heuristic, instead they are meant as mere placeholders for the assigned pair of search heuristics assigned to each NK space (e.g., For $\mathrm{NK}_{1}$, "A" species agents may be

9 assigned heuristic 1 and "B" species agents may be assigned heuristic 2; For $\mathrm{NK}_{2}$, " $\mathrm{A}$ " species agent may 10 be assigned heuristic 3 and " $\mathrm{B}$ " species agent may be assigned heuristic 4; For $\mathrm{NK}_{3}$, " $\mathrm{A}$ " species agent may 11 be assigned heuristic 1 and " $\mathrm{B}$ " species agent may be assigned heuristic 3; etc.). Each heuristic is a unique way to manipulate bits in the N-bit array that agents are initially assigned or use to "explore" the problem

13 space. These heuristics are designed to be as mutually exclusive as possible such that the odds that an A 14 species agent and a B species agent that both receive the same solution and explore the NK space to find 15 the same solution is minimal. We take inspiration from Gomez and Lazer (2019) in how they design their 16 agents.

17 - Heuristic 1 (defined in the code as agent_explore_random_0): From the N bits, a bit is randomly 18 chosen in the string and is flipped to a zero, irrespective of what its original value (i.e., a bit with 19 the value zero remains a zero).

- Heuristic 2 (defined in the code as agent_explore_random_1): From the $\mathrm{N}$ bits, a bit is randomly 21 chosen in the string and is flipped to a one, irrespective of what its original value. (i.e., a bit with 22 the value one remains a one).

23 - Heuristic 3 (defined in the code as agent_explore_flip): From the $\mathrm{N}$ bits, a bit is randomly chosen in the string and is flipped its opposite value (e.g., from 0 to 1 or 1 to 0 ). 
- Heuristic 4 (defined in the code as agent_explore_shuffle): From the $N$ bits, at least two index locations are sampled to create a substring. The agent then rotates the values of the selected index locations to the right with looping around. Once complete, the substring is inserted back into the original index locations with the new rotated values. For example, if position 3, position 5, and position 8 are chosen, the value at position 3 is moved to position 5 , the value originally at position 5 to position 8 , and the value originally at position 8 moves to position 3 . 

Local Tie Search

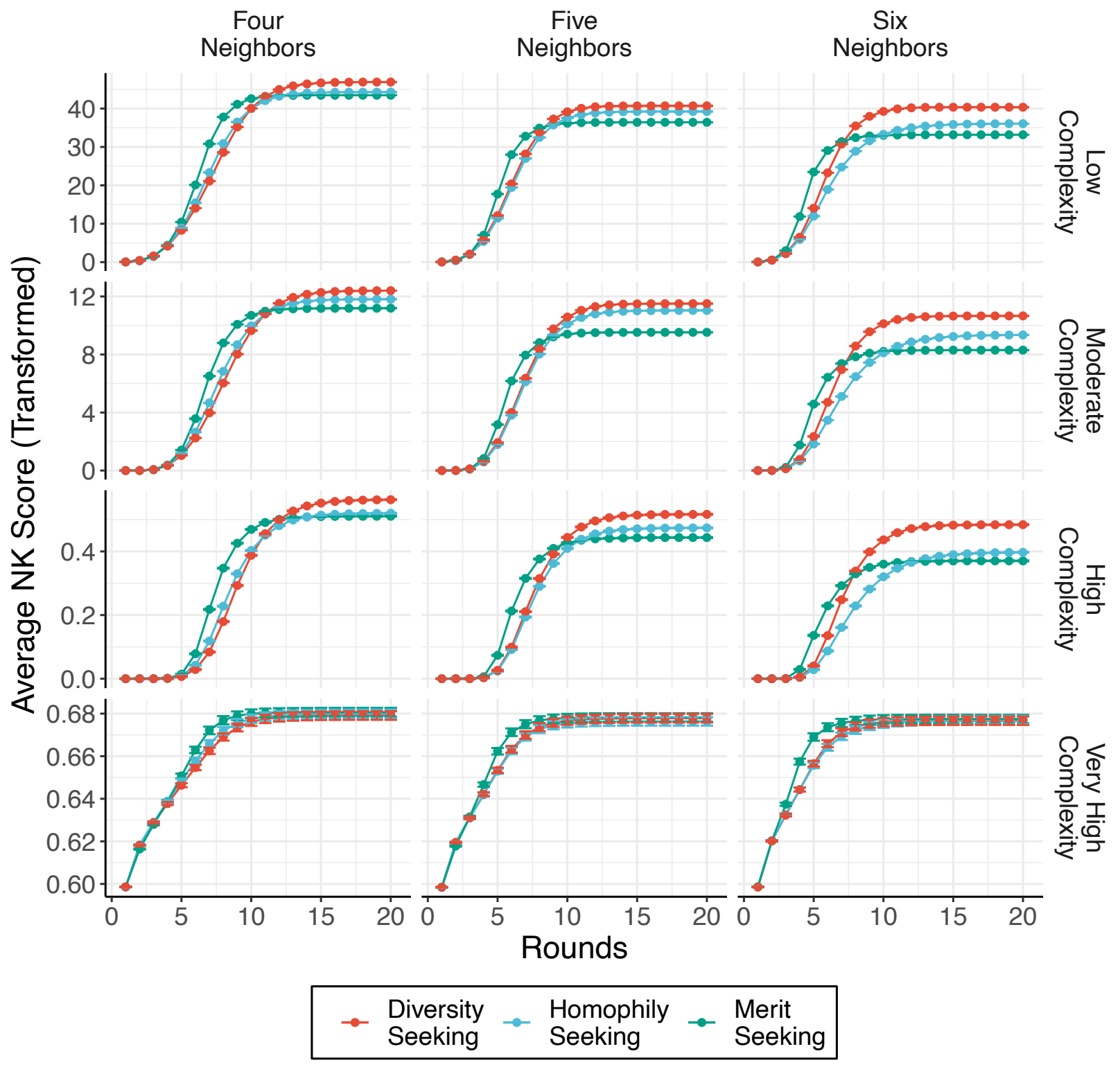

Figure B1. Averaged normalized NK scores over time for the three treatment conditions - diversity-seeking networks, homophily-seeking networks, and merit-seeking networks - across different neighbor criteria (e.g., four, five, and six, as show in the main results) and degrees of complexity in the NK space (i.e., low complexity of $K=1$, moderate complexity of $K=3$, high complexity $K=5$, as shown in the main results, and $K=10$, very high complexity). Across nearly all setups, diversity-seeking networks perform the best in the long-run and merit-seeking networks do better in the short-run, broadly consistent with what we show in the main results. 


\section{Local Tie Search}

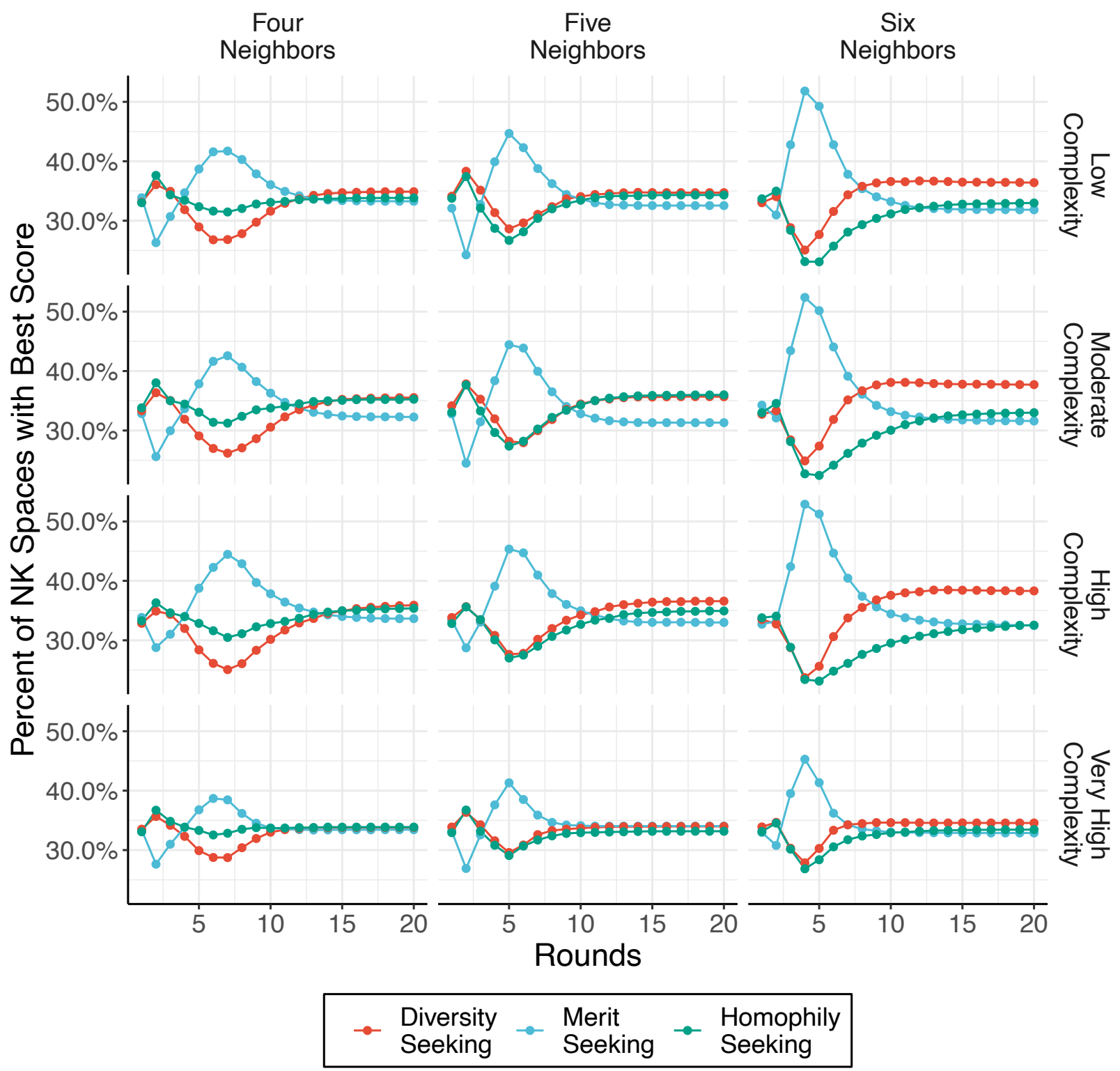

Figure B2. Percent of NK spaces with the best score among three over time for the three treatment conditions - diversity-seeking networks, homophily-seeking networks, and merit-seeking networksacross different neighbor criteria (e.g., four, five, and six, as show in the main results) and degrees of complexity in the NK space (i.e., low complexity of $K=1$, moderate complexity of $K=3$, high complexity $K=5$, as shown in the main results, and $K=10$, very high complexity). In all setups, we find a similar pattern to what we find in our main results: merit-seeking networks do well in the short-run, and diversity-seeking networks, on average, do better in the long-run. When the neighbor criterion is lowered to four, there is no benefit to diversity-seeking networks; merit-seeking networks do about the same. This also holds true for when the NK spaces are much easier $(K=1)$ to navigate or when it is too difficult to navigate $(K=10)$. 
Local Tie Search

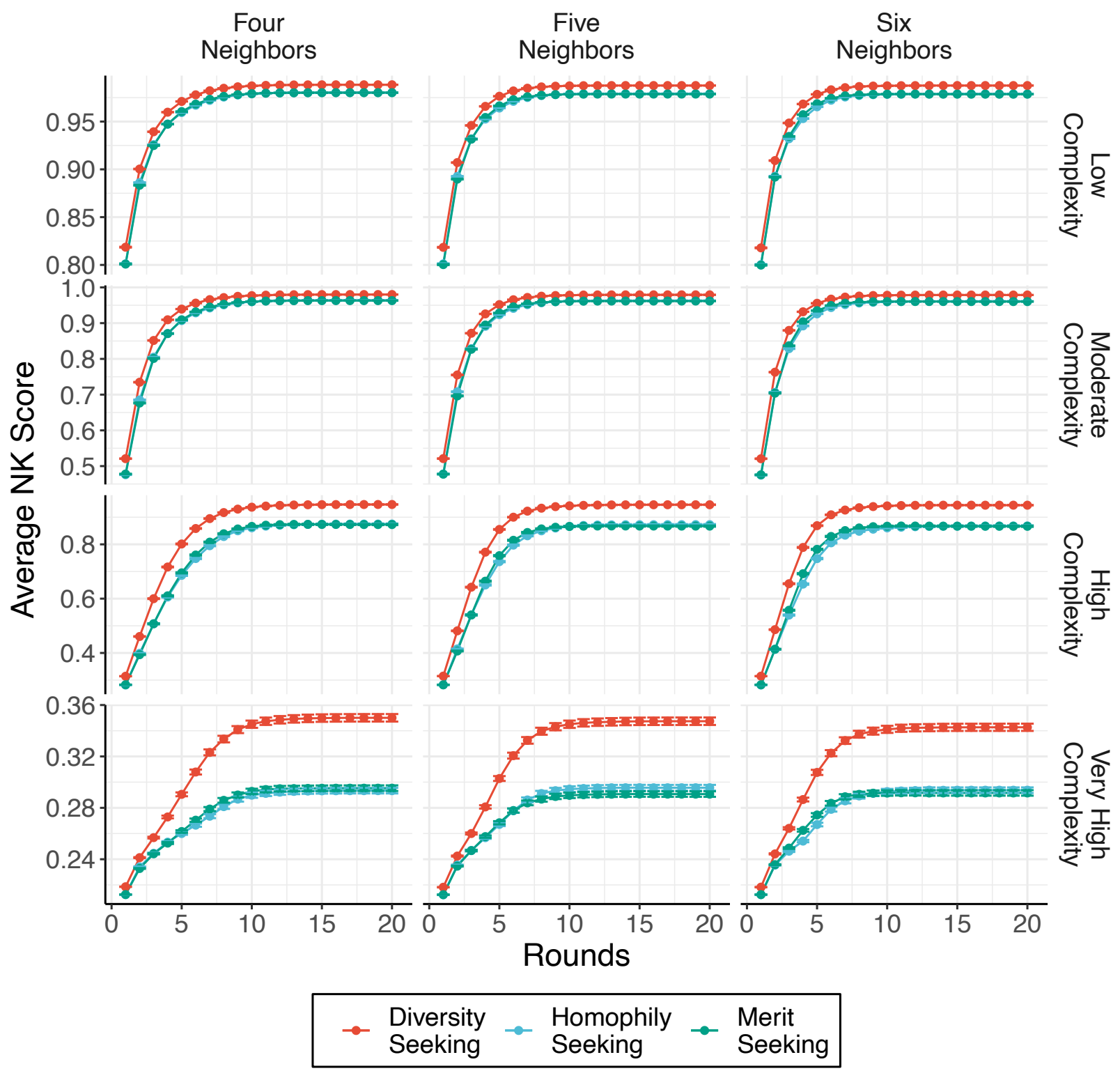

Figure B3. Average NK score of networks that find the best score among the three treatment conditionsdiversity-seeking networks, homophily-seeking networks, and merit-seeking networks - across different neighbor criteria (e.g., four, five, and six, as show in the main results) and degrees of complexity in the NK space (i.e., low complexity of $K=1$, moderate complexity of $K=3$, high complexity $K=5$, as shown in the main results, and $K=10$, very high complexity). In all settings, diverse-seeking networks consistently find the highest quality solutions. 


\section{Global Tie Search}

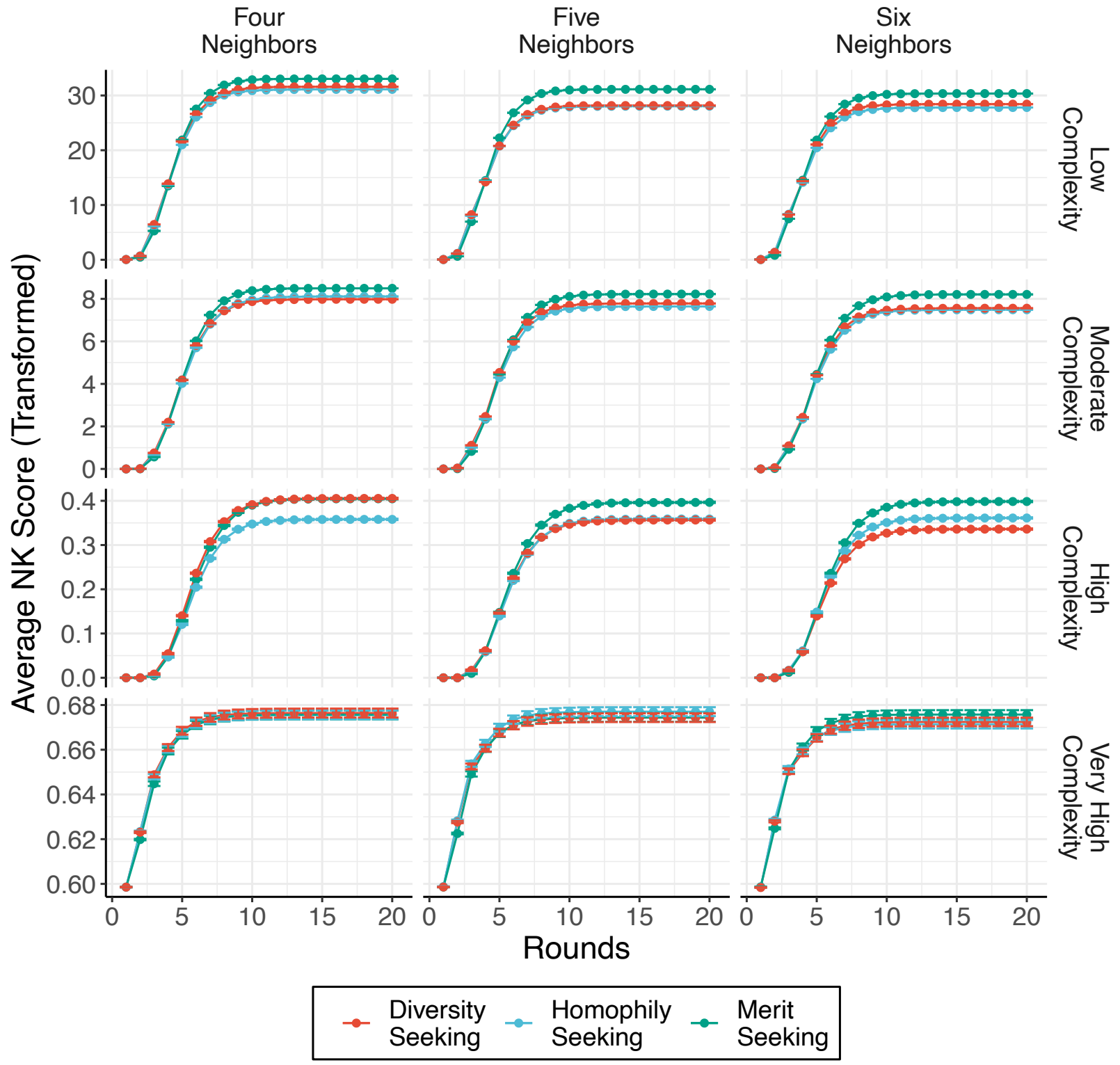

3 Figure C1. For when tie search is non-localized (i.e., global tie search), averaged normalized NK scores over time for the three treatment conditions - diversity-seeking networks, homophily-seeking networks, and merit-seeking networks - across different neighbor criteria (e.g., four, five, and six, as show in the main results) and degrees of complexity in the NK space (i.e., low complexity of $K=1$, moderate complexity of $K=3$, high complexity $K=5$, as shown in the main results, and $K=10$, very high complexity). Across conditions, merit-seeking outperforms diversity and merit-seeking networks. This is, in part, due to the wider pool agents have access to. Merit-seeking networks are able to find agents with better solutions earlier on. However, the performance of these networks are worse than their localized counterparts. 


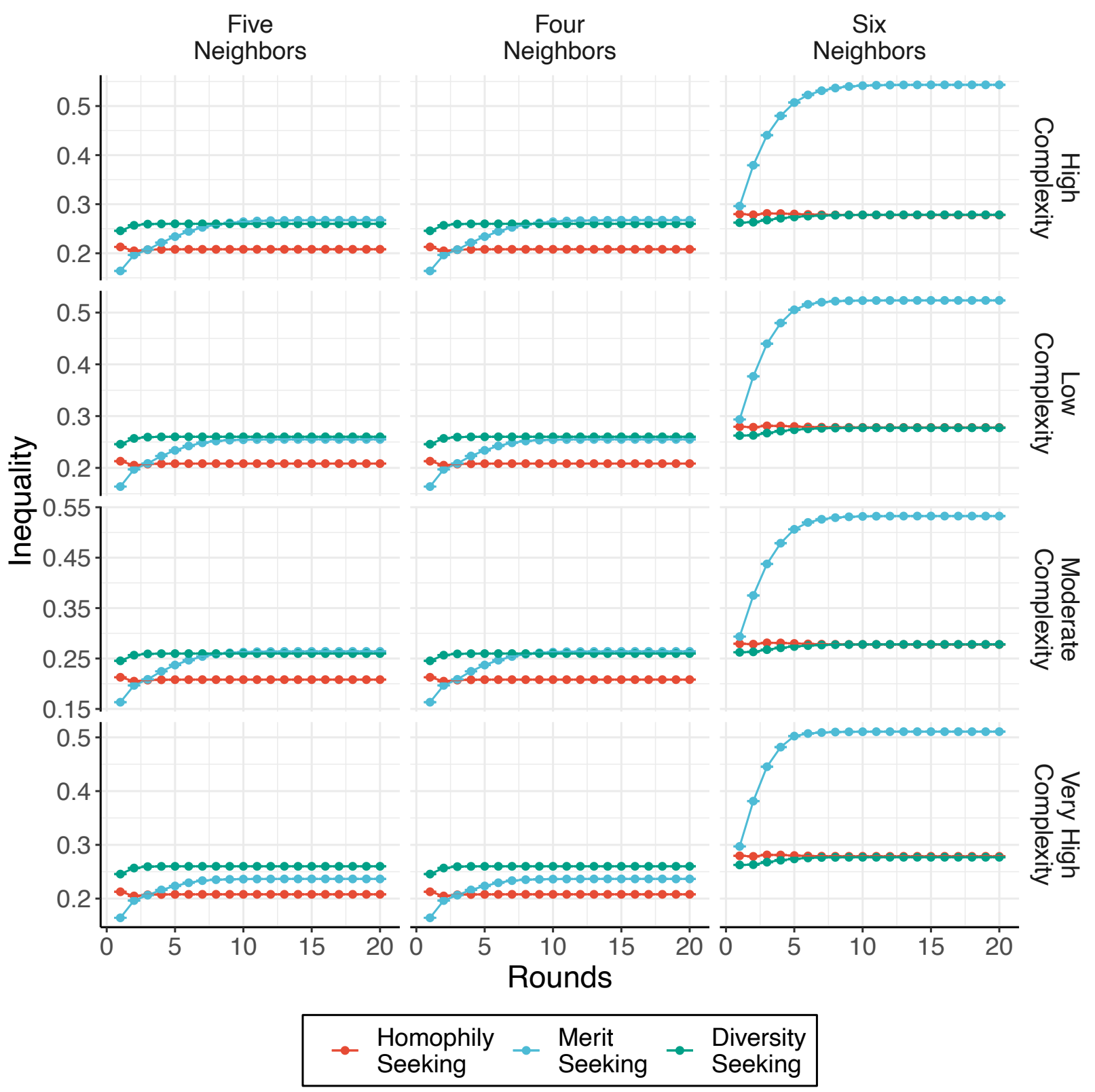

Figure D2. Network inequality, the Gini coefficient for the in-degree distribution for each of the three heuristics by round across different neighbor criteria (e.g., four, five, and six, as show in the main results) and degrees of complexity in the NK space (i.e., low complexity of $K=1$, moderate complexity of $K=3$, high complexity $K=5$, as shown in the main results, and $K=10$, very high complexity). Only when all six neighbors are considered by agents do we see any meaningful difference in inequality. Yet, across all NK spaces, the results are qualitatively the same and mirror what we present in the main paper. 


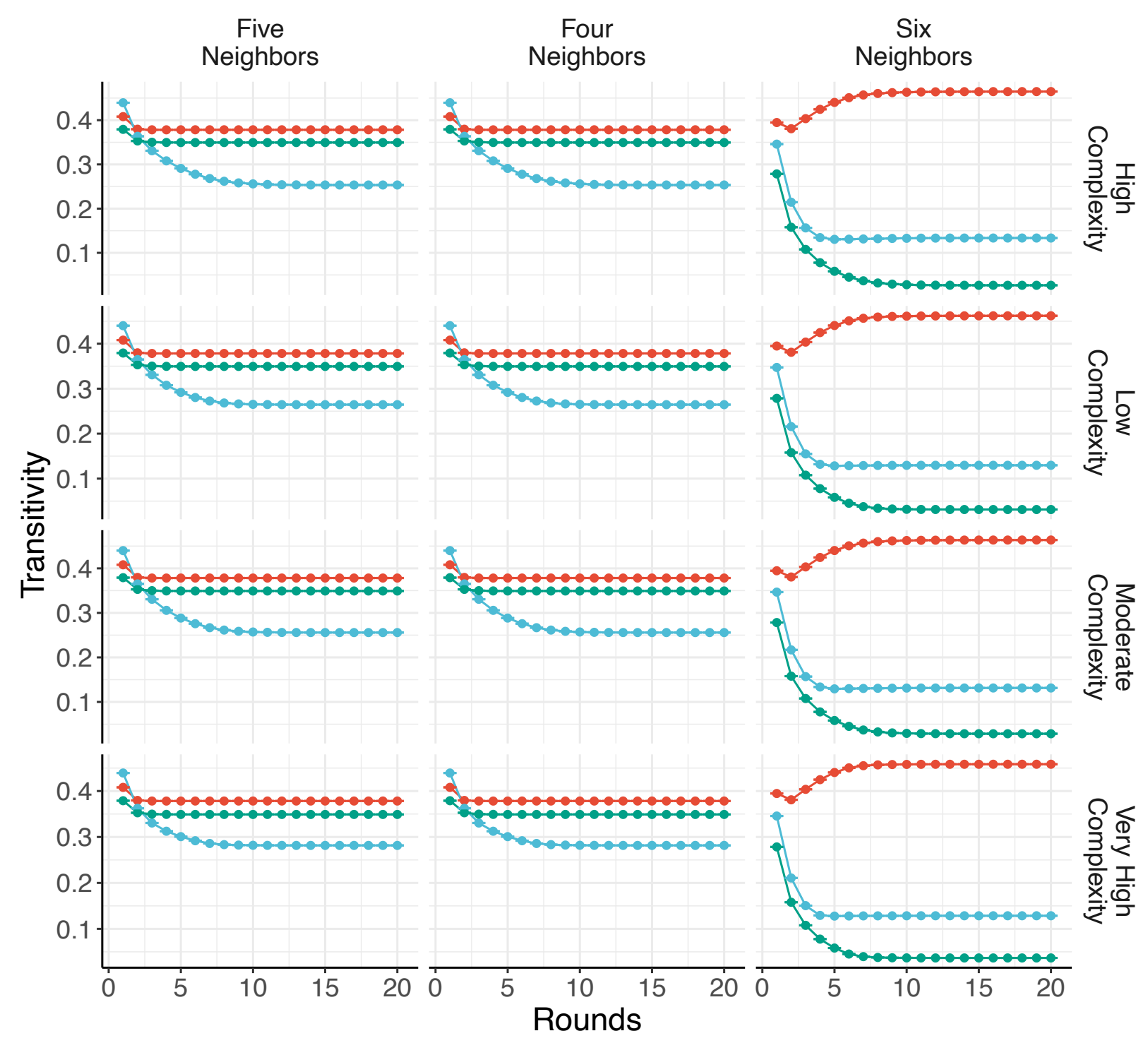

Figure D3. Network transitivity, the proportion of all possible triangles that exist in each network, for each of the three heuristics by round across different neighbor criteria (e.g., four, five, and six, as show in the main results) and degrees of complexity in the NK space (i.e., low complexity of $K=1$, moderate complexity of $K=3$, high complexity $K=5$, as shown in the main results, and $K=10$, very high complexity). Only when all six neighbors are considered by agents do we see any meaningful difference in transitivity. Yet, across 7 all NK spaces, the results are qualitatively the same and mirror what we present in the main paper. 